\title{
LOW-VELOCITY SHOCKS TRACED BY EXTENDED SiO EMISSION ALONG THE W43 RIDGES: WITNESSING THE FORMATION OF YOUNG MASSIVE CLUSTERS
}

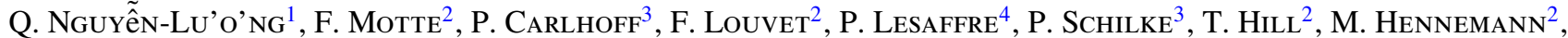

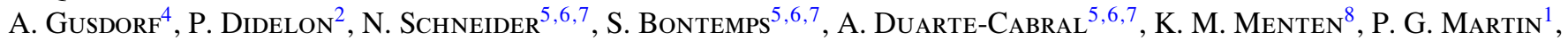 \\ F. Wyrowski ${ }^{8}$, G. Bendo ${ }^{9}$, H. Roussel ${ }^{10}$, J.-P. Bernard ${ }^{11,12}$, L. Bronfman ${ }^{13}$, T. Henning ${ }^{14}$, C. Kramer ${ }^{15}$, and F. Heitsch ${ }^{16}$ \\ ${ }^{1}$ Canadian Institute for Theoretical Astrophysics, University of Toronto, 60 St. George Street, Toronto, ON M5S 3H8, Canada; qnguyen@ cita.utoronto.ca \\ ${ }^{2}$ Laboratoire AIM Paris-Saclay, CEA/IRFU - CNRS/INSU - Université Paris Diderot, Service d'Astrophysique, \\ Bât. 709, CEA-Saclay, F-91191, Gif-sur-Yvette Cedex, France \\ ${ }^{3}$ I. Physikalisches Institut, Universität zu Köln, Zülpicher Str. 77, D-50937 Köln, Germany \\ ${ }^{4}$ ENS, LERMA, UMR 8112, CNRS, Observatoire de Paris, 24 rue Lhomond 75005 Paris, France LRA/ENS, France \\ ${ }^{5}$ Université de Bordeaux, LAB, CNRS, UMR 5804, F-33270 Floirac, France \\ ${ }^{6}$ Univ. Bordeaux, LAB, UMR 5804, F-33270, Floirac, France \\ ${ }^{7}$ CNRS, LAB, UMR 5804, F-33270, Floirac, France \\ ${ }^{8}$ Max-Planck-Institut für Radioastronomie, Auf dem Hügel 69, D-53121 Bonn, Germany \\ ${ }^{9}$ UK ALMA Regional Centre Node, Jodrell Bank Centre for Astrophysics, School of Physics and Astronomy, \\ University of Manchester, Oxford Road, Manchester M13 9PL, UK \\ ${ }^{10}$ Institut d'Astrophysique de Paris, UMR 7095 CNRS, Université Pierre \& Marie Curie, 98 bis boulevard Arago, F-75014, Paris, France \\ ${ }^{11}$ Université de Toulouse, UPS, CESR, 9 Avenue du Colonel Roche, F-31028 Toulouse Cedex 4, France \\ 12 CNRS, UMR 5187, F-31028 Toulouse, France \\ 13 Departamento de Astronomía, Universidad de Chile, Santiago, Chile \\ ${ }^{14}$ Max Planck Institute for Astronomy, Koenigstuhl 17, D-69117 Heidelberg, Germany \\ 15 Instituto Radioastronomía Milimétrica (IRAM), Av. Divina Pastora 7, Núcleo Central, E-18012 Granada, Spain \\ ${ }^{16}$ Department of Physics and Astronomy, University of North Carolina Chapel Hill, Phillips Hall, Chapel Hill, NC 27599-3255, USA \\ Received 2013 April 12; accepted 2013 July 20; published 2013 September 10
}

\begin{abstract}
The formation of high-mass stars is tightly linked to that of their parental clouds. Here, we focus on the high-density parts of W43, a molecular cloud undergoing an efficient event of star formation. Using a column density image derived from Herschel continuum maps, we identify two high-density filamentary clouds, called the W43-MM1 and W43-MM2 ridges. Both have gas masses of $2.1 \times 10^{4} M_{\odot}$ and $3.5 \times 10^{4} M_{\odot}$ above $>10^{23} \mathrm{~cm}^{-2}$ and within areas of $\sim 6$ and $\sim 14 \mathrm{pc}^{2}$, respectively. The W43-MM1 and W43-MM2 ridges are structures that are coherent in velocity and gravitationally bound, despite their large velocity dispersion measured by the $\mathrm{N}_{2} \mathrm{H}^{+}(1-0)$ lines of the W43-HERO IRAM large program. Another intriguing result is that these ridges harbor widespread $\left(\sim 10 \mathrm{pc}^{2}\right)$ bright $\mathrm{SiO}(2-1)$ emission, which we interpret to be the result of low-velocity shocks $\left(\leqslant 10 \mathrm{~km} \mathrm{~s}^{-1}\right)$. We measure a significant relationship between the $\mathrm{SiO}(2-1)$ luminosity and velocity extent and show that it distinguishes our observations from the high-velocity shocks associated with outflows. We use state-of-the-art shock models to demonstrate that a small percentage (10\%) of Si atoms in low-velocity shocks, observed initially in gas phase or in grain mantles, can explain the observed $\mathrm{SiO}$ column density in the W43 ridges. The spatial and velocity overlaps between the ridges of high-density gas and the shocked $\mathrm{SiO}$ gas suggest that ridges could be forming via colliding flows driven by gravity and accompanied by low-velocity shocks. This mechanism may be the initial conditions for the formation of young massive clusters.
\end{abstract}

Key words: H II regions - ISM: clouds - ISM: molecules - ISM: structure - stars: formation - stars: protostars

Online-only material: color figures

\section{INTRODUCTION}

Despite being an important entity in the energy budget and the cloud structure of galaxies, the origins of high-mass stars (OB-type, $>8 M_{\odot}$ ) are uncertain. An essential question is how they can gain such relatively large masses within their short protostellar lifetime $\left(10^{5}-10^{6} \mathrm{yr}\right)$. Two main theoretical explanations were proposed to answer this question. In the quasistatic view, a high degree of turbulence allows the formation of a massive dense core inside which a powerful accretion develops to build up the stellar mass (e.g., Krumholz et al. 2009; Hosokawa \& Omukai 2009). In the dynamical picture, competitive accretion and/or cloud formation generate colliding flows which then funnel gas to a large potential well (e.g., Bonnell \& Bate 2006; Heitsch \& Hartmann 2008; Inoue \& Inutsuka 2009; Hartmann et al. 2012). Dynamical signatures such as gravita- tional streamers and shearing motions at molecular-cloud scales (10-100 pc; Nguyen Luong et al. 2011b), filamentary scales (1-10 pc; Schneider et al. 2010a; Hennemann et al. 2012), and protostellar core scales (0.1-1 pc; Csengeri et al. 2011a, 2011b) have been observed. All of these studies convey the idea that the formation of high-mass stars is tightly linked to the density, kinematics, and formation of their parental cloud.

HOBYS, ${ }^{17}$ a key mapping survey with Herschel,,${ }^{18}$ is dedicated to the formation of OB-type stars within molecular complexes (Motte et al. 2010, 2012). It has shown that molecular clouds are dominated by networks of filaments, and that

\footnotetext{
17 The Herschel imaging survey of OB Young Stellar objects (HOBYS) is a Herschel key program. See http://hobys-herschel.cea.fr.

18 Herschel is an ESA space observatory with science instruments provided by European-led Principal Investigator consortia and with important participation from NASA.
} 
clusters of high-mass protostars are forming in "cloud ridges" (Hill et al. 2011; Nguyen Luong et al. 2011a; Hennemann et al. 2012). These "ridges" are high-density (equivalent $N_{\mathrm{H}_{2}}>$ $10^{23} \mathrm{~cm}^{-2}$ ), elongated cloud structures, which dominate and shape their surroundings. Clusters of massive stars are forming within these ridges, making them excellent candidate sites to host mini-starbursts, i.e., miniature and instant models of intense star formation activity (Hill et al. 2011; Nguyen Luong et al. 2011a). Schneider et al. (2012) also showed that intermediate-mass stellar clusters generally form at the junction of filaments. The density structure and star formation efficiency (SFE) of ridges suggest that they have formed through dynamical processes such as colliding flows and/or filaments merging. In the converging flows theory, ridges can be the result of these large-scale processes that sweep up and accumulate gas mass (Ballesteros-Paredes et al. 1999; Hartmann et al. 2001; Inoue \& Inutsuka 2009). Alternatively, they could be formed by the gravitational focusing of gas toward large potential wells (Hartmann \& Burkert 2007). The massive dense cores are then formed within shock-compressed ridges, which were created by shock waves from cloud-cloud collision (Inoue \& Fukui 2013).

The W43 complex lies at $6 \mathrm{kpc}$ from the Sun, at the meeting point of the Scutum-Centaurus (or Scutum-Crux) Galactic arm and the bar, a dynamically complex region where high-velocity streams could easily collide (Nguyen Luong et al. 2011b). The peculiar properties of W43 have been confirmed by the highangular resolution observations of the ${ }^{13} \mathrm{CO}(2-1)$ and $\mathrm{C}^{18} \mathrm{O}$ (2-1) lines of the W43-HERO IRAM ${ }^{19}$ large program (W43 Hera/EmiR Observations; see Carlhoff et al. 2013). Close to its center, W43-Main is undergoing a remarkably efficient episode of star formation and qualifies as a mini-starburst $\left(\mathrm{SFE} \sim 25 \%, \Sigma_{\mathrm{SFR}} \sim 1000 M_{\odot} \mathrm{yr}^{-1} \mathrm{kpc}^{-2}\right.$; Motte et al. 2003). According to the criteria of Bressert et al. (2012), the W43-MM1 and W43-MM2 clouds are indeed good candidates to form the future young massive clusters (see Ginsburg et al. 2012). Among the 15 dense cores of W43-Main (0.2 pc FWHM size and $>5 \times 10^{5} \mathrm{~cm}^{-3}$ density), three extremely massive, dense cores are potentially forming high-mass stars: W43MM1 $\left(M=3600 M_{\odot}\right)$, W43-MM2 $\left(M=1600 M_{\odot}\right)$, and W43-MM3 $\left(M=1000 M_{\odot}\right.$; Motte et al. 2003, confirmed by Bally et al. 2010). Adjacent to W43-Main is a giant $\mathrm{H}$ II region, illuminated by a cluster of Wolf-Rayet (W-R) and OB stars, emitting $\sim 10^{51}$ Lyman continuum photons per second and a farinfrared continuum luminosity of $\sim 3.5 \times 10^{6} L_{\odot}$ (Smith et al. 1978; Blum et al. 1999; Bik et al. 2005). It is not yet clear what the impact is of this starburst cluster on the W43-Main cloud located 2-10 pc away (Motte et al. 2003; Bally et al. 2010). With its special characteristics, W43 represents a type of molecular cloud complex which hosts high luminosity embedded clusters. Other well-known examples are W49 (Galván-Madrid 2013) and W51 (Bieging et al. 2010).

Theoretically, a series of shocks are expected to emerge at the swept-up edges of turbulent colliding flows as a consequence of compression and shears of material (Koyama \& Inutsuka 2000; Heitsch et al. 2008). Detecting these shocks through observation will generate the firmest evidence for the collision and merging of several gas flows. Classically, $\mathrm{SiO}$ is the diagnostic of highvelocity shocks $\left(v_{\text {shock }} \sim 20-50 \mathrm{~km} \mathrm{~s}^{-1}\right)$ in protostellar outflows

\footnotetext{
19 The W43-HERO (W43 Hera/EmiR Observation) project is an IRAM $30 \mathrm{~m}$ large program called "Origins of molecular clouds and star formation in W43," led by Frédérique Motte and Peter Schilke. The project description and data can be accessed here: http://www.iram-institute.org/EN/content-page292-7-158-240-292-0.html. IRAM is supported by INSU/CNRS (France), MPG (Germany) and IGN (Spain).
}

(Schilke et al. 1997; Gueth et al. 1998; Gusdorf et al. 2008b). It has also been proven to trace medium- to high-velocity irradiated shocks $\left(v_{\text {shock }} \sim 10-20 \mathrm{~km} \mathrm{~s}^{-1}\right.$ to $\left.v_{\text {shock }} \sim 50 \mathrm{~km} \mathrm{~s}^{-1}\right)$ from hot cores/hot corinos (Hatchell et al. 2001; Jørgensen et al. 2011), and photon-dominated regions (PDRs; e.g., Schilke et al. $2001)$, or very high velocity shocks $\left(v_{\text {shock }} \sim 100 \mathrm{~km} \mathrm{~s}^{-1}\right)$ in the Central Molecular Zone (CMZ) of our Galaxy (e.g., MartinPintado et al. 1997; Jones et al. 2012). SiO emission is also detected in the locations where protostellar outflows interact with local gas condensations (Lefloch et al. 1998). Most of the surveys performed toward high-mass protostars interpreted the $\mathrm{SiO}$ emission as solely tracing high-velocity protostellar outflows (e.g., Beuther \& Sridharan 2007; López-Sepulcre et al. 2011). When analyzing the $\mathrm{SiO}$ spectra of the Cygnus X massive dense cores, Motte et al. (2007), however, felt the necessity to decompose the line into two shocked gas components: a broad one tracing the protostellar outflow and a narrow one interpreted as arising from the hot core. More recently, Duarte-Cabral et al. (2013) revisited the question of the $\mathrm{SiO}$ line profiles in Cygnus $\mathrm{X}$ and proposed that the narrow component is most probably associated with the shocks expected at the locations of the velocity shears observed by Csengeri et al. (2011a, 2011b) at the scale of massive dense cores $(0.1 \mathrm{pc})$. Jiménez-Serra et al. (2010) have also proposed that part of the extended $\mathrm{SiO}$ emission observed along IRDC G035.39-00.33 is associated with lowvelocity shocks from colliding flows (see also Henshaw et al. 2013). This statement was confirmed, at one location of the filament, by the lack of intermediate- to high-mass protostars that could explain the $\mathrm{SiO}$ emission by shocks from a protostellar outflow or a hot core (see Nguyen Luong et al. 2011a).

In the present paper, we report the discovery of bright and widespread $\mathrm{SiO}$ emission along the W43-MM1 and W43-MM2 ridges, whose physical properties are constrained through Herschel maps, $\mathrm{N}_{2} \mathrm{H}^{+}(1-0)$ and $\mathrm{SiO}(2-1)$ spectral cubes. The paper is organized such that Section 2 describes the data employed, Section 3 characterizes the W43-MM1 and W43-MM2 cloud ridges, and Section 4 characterizes their $\mathrm{SiO}$ emission. In Section 5, we show that state-of-the-art models can create strong $\mathrm{SiO}$ emission with low-velocity shocks, and we propose that this is the origin of the $\mathrm{SiO}$ emission we observe in W43 ridges. We also propose in Section 5, that the extended $\mathrm{SiO}$ emission directly relates to the formation of ridges and discuss the implications for the subsequent high-mass star formation. We conclude and summarize our findings in Section 6.

\section{OBSERVATIONS}

To study the connection between the density and the velocity structures of W43-Main and the shock properties within this region, we used both continuum data from Herschel and $\mathrm{SiO}$, $\mathrm{N}_{2} \mathrm{H}^{+}$emission lines from the IRAM $30 \mathrm{~m}$ telescope.

\subsection{Dust Continuum Imaging with Herschel}

We used the Herschel images obtained from the HiGAL survey (Herschel Infrared Galactic Plane Survey, ObsIDs: 1342186275 and 1342186276; Molinari et al. 2010) at $70 / 160 \mu \mathrm{m}$ with PACS (Poglitsch et al. 2010) and at $250 / 350 / 500 \mu \mathrm{m}$ with SPIRE (Griffin et al. 2010). We complemented the Hi-GAL nominal-mode dataset with SPIRE 250/350/500 $\mu \mathrm{m}$ bright-mode observations from the HOBYS key program (ObsIDs: 1342239977 and 1342239978; Motte et al. 2010). The Hi-GAL data were taken in parallel mode with a fast scanning speed of $60^{\prime \prime} \mathrm{s}^{-1}$ and the HOBYS data in 
Table 1

Observational Parameters of Herschel Images

\begin{tabular}{lcrccc}
\hline \hline$\lambda(\mu \mathrm{m})$ & HPBW & $f^{\mathrm{a}}$ & \multicolumn{2}{c}{$1 \sigma \mathrm{rms}\left(\mathrm{MJy} \mathrm{sr}^{-1}\right)$} & Pixel \\
\cline { 5 - 6 } /Camera & $\left({ }^{\prime \prime}\right)$ & & Hi-GAL & HOBYS & Size $\left(^{\prime \prime}\right)$ \\
\hline 70/PACS & $5.8 \times 12.1$ & 21706 & 0.02 & $\ldots$ & 1.40 \\
160/PACS & $11.4 \times 13.4$ & 5237 & 0.08 & $\ldots$ & 2.85 \\
250/SPIRE & 18.1 & 115 & 1.00 & 0.1 & 4.50 \\
350/SPIRE & 25.2 & 60 & 1.10 & 0.2 & 6.25 \\
500/SPIRE & 36.9 & 27 & 1.20 & 0.4 & 9.00 \\
\hline
\end{tabular}

Note. ${ }^{\text {a }}$ Conversion factor from original units, Jy pixel $^{-1}$ for PACS data and $\mathrm{Jy} \mathrm{beam}^{-1}$ for SPIRE data to MJy $\mathrm{sr}^{-1}$.

\section{Table 2}

Observational Parameters for the IRAM 30 m Lines

\begin{tabular}{lccc}
\hline \hline Parameter & Unit & $\mathrm{SiO}(2-1)$ & $\mathrm{N}_{2} \mathrm{H}^{+}(1-0)$ \\
\hline Frequency & $(\mathrm{GHz})$ & 86.846 & 93.173 \\
Angular HPBW & $\left({ }^{\prime \prime}\right)$ & 31 & 28 \\
Linear HPBW & $(\mathrm{pc})$ & 0.90 & 0.81 \\
$\Delta V_{\text {res }}$ & $\left(\mathrm{km} \mathrm{s}^{-1}\right)$ & 0.67 & 0.13 \\
$3 \sigma$ rms & $\left(\mathrm{K} \mathrm{km} \mathrm{s}^{-1}\right)$ & 0.05 & 0.01 \\
$B_{\text {eff }}$ & $\ldots$ & 0.81 & 0.81 \\
$F_{\text {eff }}$ & $\ldots$ & 0.95 & 0.95 \\
\hline
\end{tabular}

SPIRE-only mode with a $30^{\prime \prime} \mathrm{s}^{-1}$ scanning speed. The raw (level-0) data of each individual scan from both PACS and SPIRE were calibrated and deglitched using $\mathrm{HIPE}^{20}$ version 10.0. The SPIRE and PACS level-1 data were then fed to version 18 of the Scanamorphos software package ${ }^{21}$ (Roussel 2012), which subtracts brightness drifts by exploiting the redundancy of observed points in the sky, masks remaining glitches, and produces maps. The observational parameters of the Herschel images and the conversion factor from the original intensity units to $\mathrm{MJy} \mathrm{sr}^{-1}$ are listed in Table 1 .

The Hi-GAL dataset covers the entire W43 molecular complex but saturation occurs around the bright $\left(\gtrsim 200 \mathrm{Jy}^{\text {beam }}{ }^{-1}\right)$ structures in the 250 and $350 \mu \mathrm{m}$ SPIRE images. The Hi-GAL saturated areas were corrected by the bright-mode HOBYS dataset. For each wavelength, we used the same grid for the HOBYS and Hi-GAL maps built by Scanamorphos. The pixels of the Hi-GAL 250 and $350 \mu \mathrm{m}$ maps with $\geqslant 150 \mathrm{Jy}^{\text {beam }}{ }^{-1}$ are then replaced by those of the HOBYS map, after the addition of offsets measured by cross-correlating unsaturated Hi-GAL and HOBYS pixels (see Appendix B). The resulting threecolor $(\mathrm{RGB}=250 \mu \mathrm{m} / 160 \mu \mathrm{m} / 70 \mu \mathrm{m})$ image is presented in Figures 1 and 8 .

\section{2. $\mathrm{SiO}$ and $\mathrm{N}_{2} \mathrm{H}^{+}$Mappings with the IRAM $30 \mathrm{~m}$}

We extracted the $\mathrm{SiO}(2-1)$ and $\mathrm{N}_{2} \mathrm{H}^{+}(1-0)$ emission lines from a $\sim 160^{\prime 2}$ mapping survey of the W43-Main mini-starburst region made at $3 \mathrm{~mm}(85-93 \mathrm{GHz})$. These observations were performed as part of the W43-HERO IRAM $30 \mathrm{~m}$ large program. Its first results are jointly presented in present paper and in Carlhoff et al. (2013). SiO (2-1) and $\mathrm{N}_{2} \mathrm{H}^{+}$(1-0) emission lines were observed with two different frequency setups of the Eight MIxer Receiver in 2010 December, 2011 January, and 2011 March. We used the low spectral resolution $\left(0.67 \mathrm{~km} \mathrm{~s}^{-1}\right)$ but large bandwidth $(8 \mathrm{GHz})$ Fast Fourier Transform Spectrometer backend for $\mathrm{SiO}$ lines and the high spectral resolution

\footnotetext{
20 HIPE is a joint development software by the Herschel Science Ground Segment Consortium, consisting of ESA, the NASA Herschel Science Center, and the HIFI, PACS, and SPIRE consortia.

21 http://www2.iap.fr/users/roussel/herschel/
}

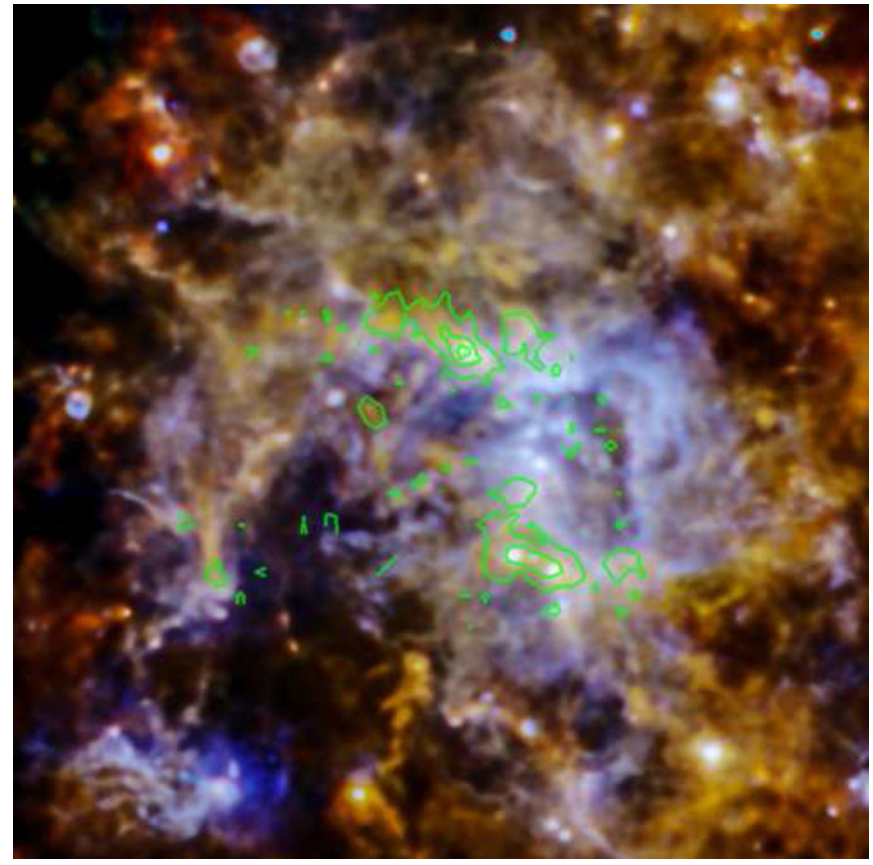

Figure 1. Composite three-color Herschel image of the W43-Main ministarburst (70 $\mu \mathrm{m}$ : blue, $160 \mu \mathrm{m}$ : green, and $250 \mu \mathrm{m}$ : red). The blue component traces $\mathrm{HII}$ and photon-dominated regions while earlier stage star-forming sites, such as cores and filaments, are traced by the red component. The contours are the integrated intensity of $\mathrm{SiO}(2-1)$, shown in Figure 5, with $3 \sigma=0.8 \mathrm{~K} \mathrm{~km} \mathrm{~s}^{-1}$ and $6 \sigma=1.6 \mathrm{~K} \mathrm{~km} \mathrm{~s}^{-1}$ levels. We note that the SiO map does not cover the full extent of the three-color image. The figure is oriented in R.A.-decl. and the area is equal to that covered in Figure 2.

(A color version of this figure is available in the online journal.)

(0.13 $\left.\mathrm{km} \mathrm{s}^{-1}\right)$ but narrow bandwidth $(80 \mathrm{MHz})$ Versatile SPectrometer Array for $\mathrm{N}_{2} \mathrm{H}^{+}$(1-0) lines. The observational parameters of the $\mathrm{SiO}$ and $\mathrm{N}_{2} \mathrm{H}^{+}$mappings are summarized in Table 2.

The maps were done using the on-the-fly mode in two perpendicular scanning directions (R.A. and decl.) and a full sampling which provides the best angular resolutions (see Table 2). Pointing, focus, and calibration observations were repeated frequently. Pointing error is less than $3^{\prime \prime}$ and calibration accuracy is within $10 \%$. The basic data reduction steps such as pointing correction, focus correction, and flux calibration were done using MIRA which is part of the GILDAS package. ${ }^{22}$ We removed a baseline from each spectrum by masking the main velocity range of W43-Main (80-100 $\mathrm{km} \mathrm{s}^{-1}$ ) and fitting a first-order polynomial to the spectrum observed over the remaining velocity ranges. We converted the unit of the data cubes from antenna temperature, $T_{\mathrm{A}^{*}}$, to main beam temperature, $T_{\mathrm{MB}}$, following the usual conversion: $T_{\mathrm{MB}}=T_{\mathrm{A}^{*}} \times F_{\text {eff }} / B_{\text {eff }}$, with main beam and forward efficiencies given in Table 2 . In the remaining parts of the paper, we used $T_{\mathrm{MB}}$ as the intensity unit. Finally, we combined the reduced spectra into gridded data cubes with a $10^{\prime \prime}$ Gaussian kernel, leading to a slightly degraded resolution (see Table 2).

\section{PROPERTIES OF CLOUD RIDGES}

In this section, we present the properties of the W43-MM1 and W43-MM2 ridges using column density and temperature images

22 GILDAS (Grenoble Image and Line Data Analysis Software) is a software package mainly developed and maintained by IRAM to reduce and analyze data obtained with the $30 \mathrm{~m}$ telescope and Plateau de Bure interferometer. See www.iram.fr/IRAMFR/GILDAS 

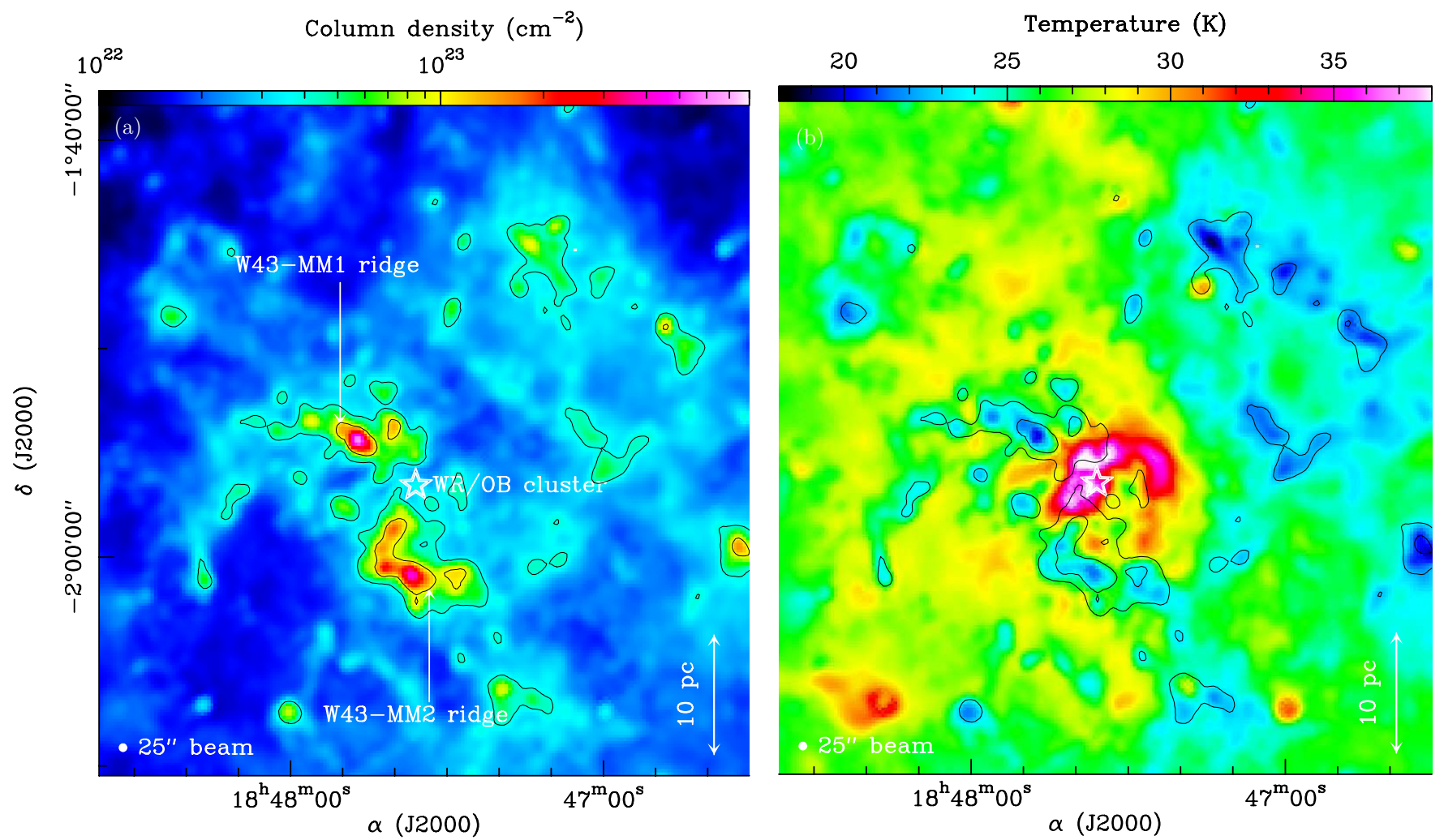

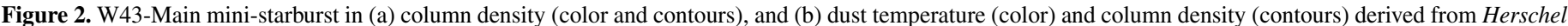

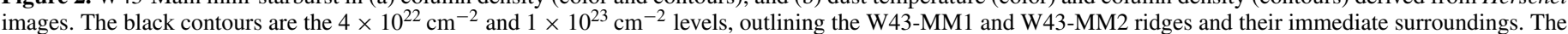
star symbol pinpoints the W-R/OB star cluster responsible for a giant $\mathrm{H}$ II region seen in (b) as a heated bubble.

(A color version of this figure is available in the online journal.)

derived from Herschel maps (Section 3.1), and the velocity structure traced by $\mathrm{N}_{2} \mathrm{H}^{+}$emission lines (Section 3.2).

\subsection{Density Structure}

Using three of the four longest wavelengths of Herschel (160-350 $\mu \mathrm{m})$, we derived the total (gas+dust) column density $\left(N_{\mathrm{H}_{2}}\right)$ and average dust temperature maps of W43-Main with an angular resolution of $25^{\prime \prime}$, which is that of the $350 \mu \mathrm{m}$ map. Following the procedure fully described in Hill et al. (2011, 2012), we fitted pixel-by-pixel spectral energy distributions (SEDs) with modified blackbody models. We used a dust opacity law similar to that of Hildebrand (1983) but with $\beta=2$ instead of $\beta=1$ and assumed a gas-to-dust ratio of 100: $\kappa_{\nu}=0.1 \times(300 \mu \mathrm{m} / \lambda)^{2} \mathrm{~cm}^{2} \mathrm{~g}^{-1}$. Given the high quality of the Herschel data, a robust fit can be done, even when dropping the $500 \mu \mathrm{m}$ data point. The column density values are only changed by a factor of less than $10 \%$ in region with $N_{\mathrm{H}_{2}}>10^{22} \mathrm{~cm}^{-2}$ (see Hill et al. 2012). Changing $\beta$ from 2 to 1.5 would globally lower the column density values by $\sim 20 \%$. Carlhoff et al. (2013) made a detailed comparison of Herschel versus CO column density maps and showed that they do agree with each other to within a factor of two for intermediate-density clouds.

The column density and temperature maps of W43-Main and the entire W43 molecular complex are plotted in Figures 2 and 9. The noise levels, estimated over areas with minimum intensity variations, are $\sigma \sim 1.5 \times 10^{21} \mathrm{~cm}^{-2}$ and $\sim 1.5 \mathrm{~K}$. The average column density and temperature found toward W43-Main are $\sim 2.5 \times 10^{22} \mathrm{~cm}^{-2}\left(A_{\mathrm{V}} \sim 25 \mathrm{mag}\right)$ and $\sim 26 \mathrm{~K}$. The dominating structure of Figure 2 is the cold and dense Z-shaped filament discussed in Motte et al. (2003), which reaches column densities up to $\sim 6.6 \times 10^{23} \mathrm{~cm}^{-2}$ and tem- peratures down to $\sim 19 \mathrm{~K}$. It contains two clouds with $N_{\mathrm{H}_{2}}$ $>10^{23} \mathrm{~cm}^{-2}$, i.e., above the column density requirement of ridges as defined by Hill et al. (2011). They host the W43MM1 and W43-MM2 massive dense cores (Motte et al. 2003) and are hereafter denoted as the W43-MM1 and W43-MM2 ridges. These ridges stand out from their environment at a column density level of $\sim 4 \times 10^{22} \mathrm{~cm}^{-2}$, inside which we defined their immediate surroundings (see Figure 2(a)). Between these ridges lies a region with higher temperature $(T>22 \mathrm{~K})$ and lower column density $N_{\mathrm{H}_{2}} \sim 3-7 \times 10^{22} \mathrm{~cm}^{-2}$ (see Figure 2). This hot bubble of gas is produced by the strong UV radiation from the massive stars within the W-R/OB cluster associated with W43-Main (star symbol in Figure 2).

We used the $N_{\mathrm{H}_{2}}=10^{23} \mathrm{~cm}^{-2}$ and $4 \times 10^{22} \mathrm{~cm}^{-2}$ contours of Figure 2(a) to measure the areas, $A$, and estimate the rough lengths, $l$, of the W43-MM1 and W43-MM2 ridges and their immediate surroundings. We assumed they are well represented by a cylindrical geometry to calculate their radii, $r=A /(2 l)$, and volumes, $V=\pi l r^{2}$. We used the $N_{\mathrm{H}_{2}}=4 \times 10^{22} \mathrm{~cm}^{-2}$ value as background emission in measuring the column density averaged over the W43-MM1 and W43-MM2 ridges, and their immediate surroundings. The mean column density after background subtraction, $\left\langle N_{\mathrm{H}_{2}}\right\rangle$, translates into mass, volume number density, and mass surface density $\left(M, n_{\mathrm{H}_{2}}, \Sigma_{S}\right)$ by the relations:

$$
\begin{gathered}
M=\mu m_{\mathrm{H}}\left\langle N_{\mathrm{H}_{2}}\right\rangle A \simeq 1853 M_{\odot} \times \frac{\left\langle N_{\mathrm{H}_{2}}\right\rangle}{10^{23} \mathrm{~cm}^{-2}} \times \frac{A}{1 \mathrm{pc}^{2}}, \\
n_{\mathrm{H}_{2}}=\frac{M}{V} \simeq 22450 \mathrm{~cm}^{-3} \times \frac{\left\langle N_{\mathrm{H}_{2}}\right\rangle}{10^{23} \mathrm{~cm}^{-2}} \times\left(\frac{r}{1 \mathrm{pc}}\right)^{-1},
\end{gathered}
$$


Table 3

Basic Characteristics of the W43 Ridges Derived from Herschel Images, $\mathrm{N}_{2} \mathrm{H}^{+}(1-0)$, and SiO (2-1) and Line Mappings

\begin{tabular}{|c|c|c|c|c|c|c|c|c|c|c|c|c|}
\hline Cloud Structure & $\begin{array}{c}A \\
\left(\mathrm{pc}^{2}\right) \\
(2)\end{array}$ & $\begin{array}{c}l \\
(\mathrm{pc}) \\
(3)\end{array}$ & $\begin{array}{l}r^{\mathrm{a}} \\
(\mathrm{pc}) \\
(4)\end{array}$ & $\begin{array}{c}V^{\mathrm{a}} \\
\left(\mathrm{pc}^{3}\right) \\
(5)\end{array}$ & $\begin{array}{c}T \\
(\mathrm{~K}) \\
(6)\end{array}$ & $\begin{array}{c}\left\langle N_{\mathrm{H}_{2}}\right\rangle^{\mathrm{b}} \\
\left(\mathrm{cm}^{-2}\right) \\
(7)\end{array}$ & $\begin{array}{c}M^{\mathrm{b}} \\
\left(M_{\odot}\right) \\
(8)\end{array}$ & $\begin{array}{c}n_{\mathrm{H}_{2}}{ }^{\mathrm{a}, \mathrm{b}} \\
\left(\mathrm{cm}^{-3}\right) \\
(9)\end{array}$ & $\begin{array}{c}\Sigma_{S} \mathrm{a}, \mathrm{b} \\
\left(M_{\odot} \mathrm{pc}^{-2}\right) \\
(10)\end{array}$ & $\begin{array}{c}M_{\mathrm{vir}}{ }^{\mathrm{c}} \\
\left(M_{\odot}\right) \\
(11)\end{array}$ & $\begin{array}{l}\frac{M}{M_{\mathrm{vir}} \mathrm{c}} \\
(12)\end{array}$ & $\begin{array}{c}L_{\mathrm{SiO}}^{\mathrm{total}} \\
\left(\mathrm{K} \mathrm{km} \mathrm{s}^{-1}\right. \\
\left.\mathrm{kpc}^{2}\right)(13)\end{array}$ \\
\hline \multicolumn{13}{|c|}{ The ridges $\left(N_{\mathrm{H}_{2}}>10^{23} \mathrm{~cm}^{-2}\right)$} \\
\hline W43-MM1 ridge & 6 & 4.3 & 0.7 & 6.6 & $19-27$ & $1.9 \times 10^{23}$ & 21000 & $6.0 \times 10^{4}$ & 3500 & 3700 & 5.5 & $1.0 \times 10^{4}$ \\
\hline W43-MM2 ridge & 14 & 8.8 & 0.8 & 17.6 & $21-28$ & $1.2 \times 10^{23}$ & 35000 & $3.4 \times 10^{4}$ & 2300 & 11000 & 2.9 & $1.0 \times 10^{4}$ \\
\hline $\mathrm{DR} 21^{\mathrm{d}}$ ridge & 2.3 & 4.0 & 0.3 & 1.1 & $14-25$ & $2.3 \times 10^{23}$ & 9800 & $1.7 \times 10^{5}$ & 4300 & $\cdots$ & $\cdots$ & \\
\hline \multicolumn{13}{|c|}{ The ridge and its immediate surrounding $\left(N_{\mathrm{H}_{2}}>4 \times 10^{22} \mathrm{~cm}^{-2}\right)$} \\
\hline W43-MM1 sur & 43 & 11.9 & 1.8 & 121.5 & $19-40$ & $4.4 \times 10^{22}$ & 32000 & $5.5 \times 10^{3}$ & 830 & 58000 & 0.6 & $2.0 \times 10^{4}$ \\
\hline W43-MM2 sur & 62 & 13.5 & 2.3 & 223.9 & $21-38$ & $4.6 \times 10^{22}$ & 53000 & $4.5 \times 10^{3}$ & 900 & 120000 & 0.5 & $2.0 \times 10^{4}$ \\
\hline DR2 $1^{\mathrm{d}}$ sur & 6.0 & 5.8 & 0.5 & 4.6 & $14-25$ & $1.1 \times 10^{23}$ & 12200 & $5.1 \times 10^{4}$ & 2000 & $\ldots$ & $\cdots$ & $\ldots$ \\
\hline
\end{tabular}

Notes.

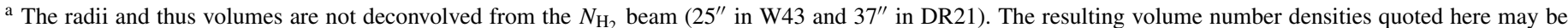

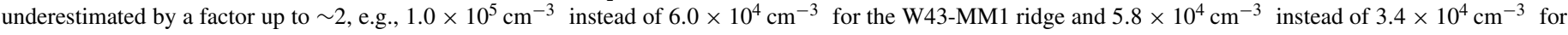
the $\mathrm{W} 43-\mathrm{MM} 2$ ridge.

b Properties measured after subtracting the common surrounding column density background of $N_{\mathrm{H}_{2} \text {, sur }}^{\text {backround }}=4 \times 10^{22} \mathrm{~cm}^{-2}$.

${ }^{c}$ Mass estimated from Column 2 and the median velocity dispersion of $\mathrm{N}_{2} \mathrm{H}^{+}(1-0)$ lines given in Table 4 .

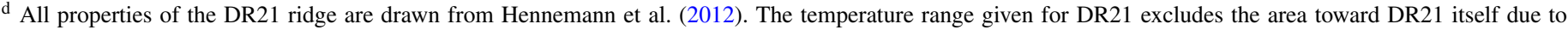
saturation, and where we expect higher dust temperatures.

$$
\Sigma_{S}=\mu m_{\mathrm{H}}\left\langle N_{\mathrm{H}_{2}}\right\rangle \simeq 1853 M_{\odot} \mathrm{pc}^{-2} \times \frac{\left\langle N_{\mathrm{H}_{2}}\right\rangle}{10^{23} \mathrm{~cm}^{-2}},
$$

where $\mu=2.33$ is the mean molecular weight and $m_{\mathrm{H}}$ is the hydrogen mass. Table 3 lists all of the characteristics derived from the Herschel column density and temperature images. We also list the properties of the DR21 ridge, drawn from Hennemann et al. (2012) with the same background subtractions, to allow a comparison with this prototypical ridge of the HOBYS survey.

Both the W43-MM1 and W43-MM2 ridges have higher temperatures than the DR21 ridge, due to their proximity to the W-R/OB cluster and a higher background of low-density, hightemperature cloud impacting the SED fits. The mean column densities of the W43-MM1 and W43-MM2 ridges are similar and are also similar to that of the DR21. For both the cloud ridges and their immediate surroundings, the masses measured for W43-MM1 and W43-MM2 are two to four times higher than the corresponding ones of DR21. This is a natural consequence of the mass being integrated over a larger area since the W43MM1 and W43-MM2 ridges and surroundings are a few times larger than the DR21 ridge. This also explains the lower values of volume number density and mass surface density, by factors of 3-10 and 1-2, measured in the W43-MM1 and the W43MM2 ridges when compared with the DR21 ridge (see Table 3). One should, however, remember that DR21 is characterized with three times higher linear spatial resolution than W43 $(0.25 \mathrm{pc}$ versus $0.7 \mathrm{pc})$. With a similar resolution, the $\left\langle N_{\mathrm{H}_{2}}\right\rangle$ value measured for W43-MM1 would increase and the cloud volume would decrease, thus, increasing the estimated volume number density by a factor of a few (see Table 3 for a tentative deconvolution of $n_{\mathrm{H}_{2}}$ ).

\subsection{Velocity Structure}

The integrated intensity map of the $\mathrm{N}_{2} \mathrm{H}^{+}(1-0)$ emission observed toward the W43-Main mini-starburst, as well as spectra at a few selected locations, are shown in Figure 3. The $\mathrm{N}_{2} \mathrm{H}^{+}(1-0)$ lines are integrated from 80 to $110 \mathrm{~km} \mathrm{~s}^{-1}$, which is the velocity range of W43-Main as defined in Nguyen Luong et al. (2011b). The selected positions are paving the
W43-MM1 and W43-MM2 ridges and have clear SiO peaks (see Figure 5). They are coincident or close to the massive dense cores identified by Motte et al. (2003) and whose names are used here for discussion (see Figures 3, 5, and Table 4). With a critical density of $\sim 4 \times 10^{5} \mathrm{~cm}^{-3}, \mathrm{~N}_{2} \mathrm{H}^{+}(1-0)$ is a good tracer of the cold dense gas in the W43-MM1 and W43-MM2 ridges, as well as their massive dense cores.

The $\mathrm{N}_{2} \mathrm{H}^{+}$(1-0) line splits into seven hyperfine structure components. We thus fitted the observed $\mathrm{N}_{2} \mathrm{H}^{+}(1-0)$ spectra by seven Gaussians, whose frequency spacings and relative line strengths are theoretically determined and cataloged in splata$\operatorname{logue}^{23}$ (see Figure 3 and Table 4). Considering the noise level in the spectra and the high probability for $\sim 0.9 \mathrm{pc}$ beams to contain several cloud components, the multiple hyperfine structure component fits to the $\mathrm{N}_{2} \mathrm{H}^{+}(1-0)$ spectra are acceptable at all locations. The derived optical depths range from 0.1 to 0.26 , meaning that $\mathrm{N}_{2} \mathrm{H}^{+}(1-0)$ emission is optically thin and traces all material along the line of sight. The W43-MM1 and W43MM2 ridges have their $V_{\mathrm{LSR}}$ spanning ranges of $94-97 \mathrm{~km} \mathrm{~s}^{-1}$ and $88-95 \mathrm{~km} \mathrm{~s}^{-1}$, respectively. At the 10 locations except W43MM1, the $\mathrm{N}_{2} \mathrm{H}^{+} V_{\mathrm{LSR}}$ are only $\leqslant 1 \mathrm{~km} \mathrm{~s}^{-1}$ different from the values determined for $\mathrm{H}^{13} \mathrm{CO}^{+}$(3-2) lines (Motte et al. 2003), it is $\sim 2 \mathrm{~km} \mathrm{~s}^{-1}$ higher for the W43-MM1 dense core.

We used the volume $V=(4 / 3) \pi r_{\mathrm{sph}}^{3}$ of the W43-MM1 and W43-MM2 ridges and the median velocity dispersion of their $\mathrm{N}_{2} \mathrm{H}^{+}$lines, $\sigma_{\mathrm{N}_{2} \mathrm{H}^{+}}$, given in Tables 3 and 4 to calculate their virial mass, $M_{\mathrm{vir}}$, according to:

$$
M_{\mathrm{vir}}=3 \times \frac{r_{\mathrm{sph}} \sigma_{\mathrm{tot}}^{2}}{G}
$$

where $G$ is the gravitational constant and $\sigma_{\text {tot }}$ is the total (thermal + nonthermal) velocity dispersion. The latter is calculated as

$$
\sigma_{\mathrm{tot}}=\sqrt{\sigma_{\mathrm{T}}^{2}+\sigma_{\mathrm{NT}}^{2}}=\sqrt{\sigma_{\mathrm{N}_{2} \mathrm{H}^{+}}+\frac{k T}{m_{\mathrm{H}}}\left(\frac{1}{\mu}-\frac{1}{\mu_{\mathrm{N}_{2} \mathrm{H}^{+}}}\right)}
$$

\footnotetext{
23 The splatalogue is a catalog of spectroscopic information of astronomical spectral lines which can be accessed from www.splatalogue.net.
} 

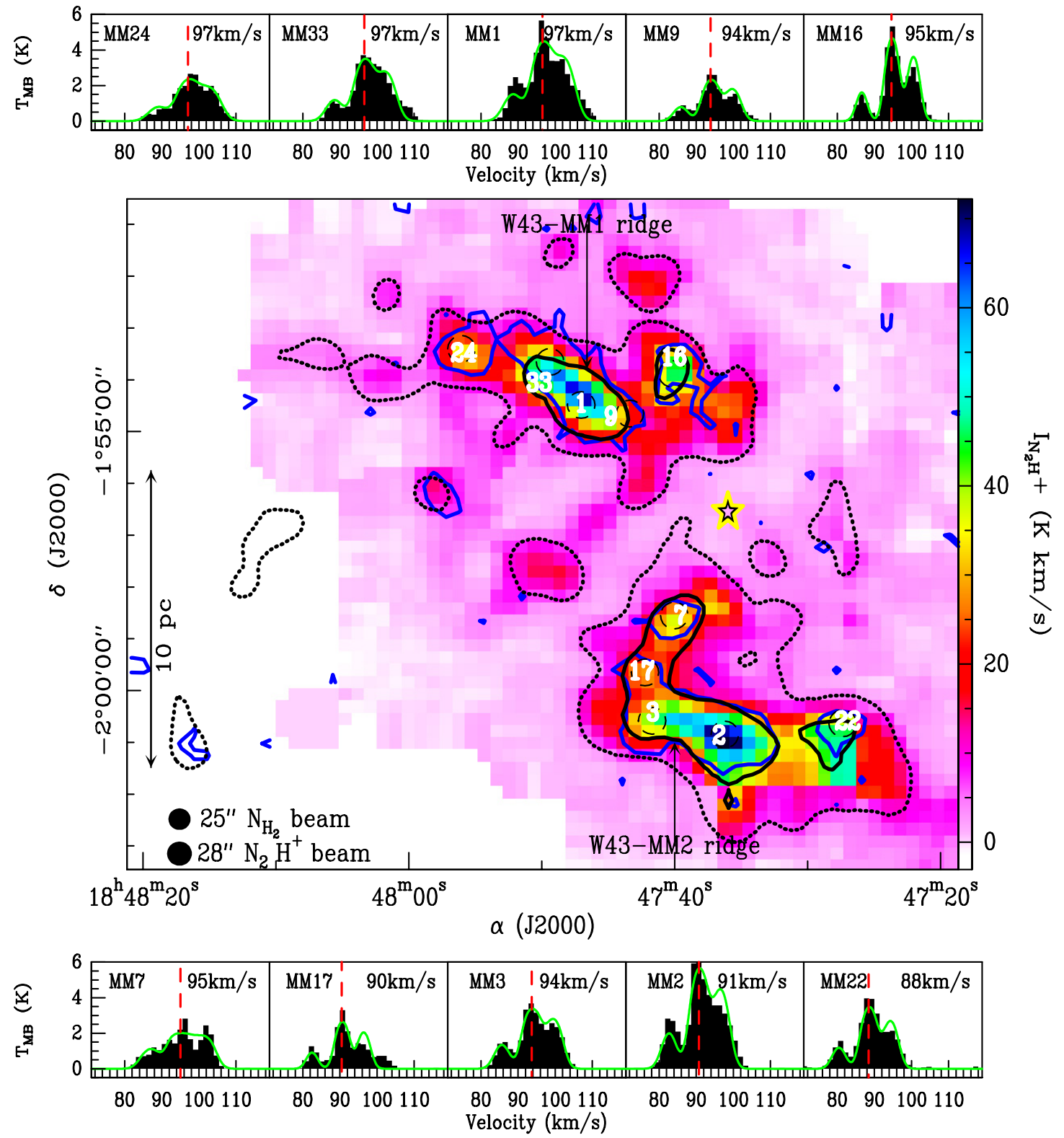

Figure 3. $\mathrm{N}_{2} \mathrm{H}^{+}(1-0)$ integrated intensity (color image), Herschel column density (black contours), and SiO (2-1) integrated intensity (blue contour) maps of W43-Main. The range of integration for both lines is $80-110 \mathrm{~km} \mathrm{~s}^{-1}$ and the $3 \sigma$ level of the $\mathrm{N}_{2} \mathrm{H}^{+}(1-0)$ integrated map is $\sim 4 \mathrm{~K} \mathrm{~km} \mathrm{~s}{ }^{-1}$. The dotted and solid contours are the $4 \times 10^{22} \mathrm{~cm}^{-2}$ and $1 \times 10^{23} \mathrm{~cm}^{-2}$ Herschel column density levels, outlining the ridges boundary and their surroundings. The blue contours indicate the regions with $\mathrm{SiO}$ emission detected above the $3 \sigma=0.8 \mathrm{~K} \mathrm{~km} \mathrm{~s}^{-1}$ level (see Figure 5). The star symbol pinpoints the W-R/OB stars cluster. $\mathrm{N}_{2} \mathrm{H}^{+}(1-0)$ spectra, measured at beam locations outlined by dashed circles. and labeled in white numbers (according to the massive dense cores numbering of Motte et al. 2003), are plotted as insets where black histograms are the measured spectra. The green curves represent the hyperfine structure fits to the spectra (see the text), and red dashed lines mark the sources peak velocity derived from the fits.

(A color version of this figure is available in the online journal.)

where $k$ is the Boltzmann constant, $m_{\mathrm{H}}$ is the hydrogen mass, $\mu=2.33$ and $\mu_{\mathrm{N}_{2} \mathrm{H}^{+}}=29$ are the mean and $\mathrm{N}_{2} \mathrm{H}^{+}$ molecular weights. We assumed that $T$ is close to the dust temperature measured in the ridges to be $\sim 23 \mathrm{~K}$. This calculation only provides approximated $M_{\text {vir }}$ values for the W43-MM1 and W43-MM2 ridges since they are better represented by cylinders than spheres and are embedded within backgrounds whose $\mathrm{N}_{2} \mathrm{H}^{+}$emission is not removed here. The unusually large virial ratios measured, $M / M_{\mathrm{vir}} \sim 5.5$ and 2.9 , indicate that, despite their high level of turbulence, the W43-MM1 and W43-MM2 ridges are most probably gravitationally bound cloud structures. The immediate surroundings, however, have much smaller virial ratios, 0.6 and 0.5 , and in- dicate that they are just at the limit to be gravitationally bound which requires $M / M_{\mathrm{vir}}>0.5$. Since $V_{\mathrm{LSR}}$ velocities in the W43MM1 and W43-MM2 ridges vary within $5 \mathrm{~km} \mathrm{~s}^{-1}$ ranges, which are close to the measured line widths, and since the virial ratio suggest boundedness/collapse, W43-MM1 and W43-MM2 ridges can be considered as coherent structures in both space and velocity.

\section{SiO EMISSION RESULT AND ANALYSIS}

\subsection{SiO Spectra and Integrated Intensity Map}

In Figure 4, we plot the accumulated spectrum representing the $\mathrm{SiO}(2-1)$ emission integrated over the entire W43-Main 
Table 4

Kinematics Overview of a Few Positions within the W43-MM1 and W43-MM2 Ridges and their Mean Characteristics

\begin{tabular}{|c|c|c|c|c|c|c|c|c|c|c|}
\hline \multirow{2}{*}{$\begin{array}{l}\text { Position/ } \\
\text { Name }\end{array}$} & \multicolumn{5}{|c|}{$\mathrm{N}_{2} \mathrm{H}^{+}(1-0)$} & \multicolumn{3}{|c|}{$\mathrm{SiO}(2-1)$} & \multirow{3}{*}{$\begin{array}{c}\sigma_{1 \mathrm{D}}{ }^{\mathrm{d}} \\
\left(\mathrm{km} \mathrm{s}^{-1}\right) \\
(10)\end{array}$} & \multirow{3}{*}{$\begin{array}{c}\Delta_{V_{\mathrm{LSR}}} \mathrm{c} \\
(11)\end{array}$} \\
\hline & $\begin{array}{c}T_{\mathrm{N}_{2} \mathrm{H}^{+}}{ }^{\mathrm{a}} \\
(\mathrm{K})\end{array}$ & $\begin{array}{c}V_{\mathrm{LSR}^{\mathrm{a}}} \\
\left(\mathrm{km} \mathrm{s}^{-1}\right)\end{array}$ & $\begin{array}{c}\Delta V_{\mathrm{FWHM}^{\mathrm{a}}} \\
\left(\mathrm{km} \mathrm{s}^{-1}\right)\end{array}$ & $\begin{array}{c}\sigma_{1 \mathrm{D}}^{\mathrm{d}} \\
\left(\mathrm{km} \mathrm{s}^{-1}\right)\end{array}$ & $\begin{array}{c}\tau_{\text {main }}{ }^{\mathrm{a}} \\
\left(\mathrm{K} \mathrm{km} \mathrm{s}^{-1}\right)\end{array}$ & $\underset{\left(\mathrm{km} \mathrm{s}^{-1}\right)}{\int T d v^{\mathrm{b}}}$ & $\begin{array}{c}V_{\mathrm{LSR}^{\mathrm{b}}} \\
\left(\mathrm{km} \mathrm{s}^{-1}\right)\end{array}$ & $\begin{array}{c}\Delta V_{\mathrm{FWHM}}{ }^{\mathrm{b}} \\
\left(\mathrm{km} \mathrm{s}^{-1}\right)\end{array}$ & & \\
\hline (1) & (2) & (3) & (4) & (5) & (6) & (7) & (8) & (9) & & \\
\hline \multicolumn{11}{|c|}{ W43-MM1 Ridge } \\
\hline W43-MM24 & $0.30 \pm 0.01$ & $97.1 \pm 0.03$ & $5.8 \pm 0.05$ & $2.5 \pm 0.02$ & $0.10 \pm 0.01$ & $2.04 \pm 0.15$ & $98.4 \pm 0.44$ & $12.2 \pm 1.08$ & $5.2 \pm 0.46$ & $-1.3 \pm 0.44$ \\
\hline W43-MM33 & $0.45 \pm 0.01$ & $96.6 \pm 0.11$ & $5.1 \pm 0.18$ & $2.1 \pm 0.08$ & $0.10 \pm 0.01$ & $3.37 \pm 0.10$ & $97.9 \pm 0.09$ & $6.8 \pm 0.22$ & $2.9 \pm 0.09$ & $-1.3 \pm 0.14$ \\
\hline W43-MM1 & $0.58 \pm 0.01$ & $96.6 \pm 0.08$ & $5.5 \pm 0.18$ & $2.3 \pm 0.08$ & $0.10 \pm 0.03$ & $7.00 \pm 0.05$ & $98.3 \pm 0.08$ & $7.8 \pm 0.22$ & $3.3 \pm 0.09$ & $-1.7 \pm 0.11$ \\
\hline W43-MM9 & $0.62 \pm 0.01$ & $94.6 \pm 0.01$ & $3.4 \pm 0.01$ & $1.4 \pm 0.01$ & $0.10 \pm 0.01$ & $1.20 \pm 0.11$ & $94.0 \pm 0.29$ & $5.9 \pm 0.83$ & $2.5 \pm 0.35$ & $0.6 \pm 0.29$ \\
\hline W43-MM16 & $0.31 \pm 0.01$ & $93.9 \pm 0.03$ & $4.3 \pm 0.05$ & $1.8 \pm 0.02$ & $0.10 \pm 0.01$ & $0.77 \pm 0.08$ & $94.1 \pm 0.27$ & $6.5 \pm 0.85$ & $2.8 \pm 0.36$ & $-0.2 \pm 0.3$ \\
\hline W43-MM1 ridge & $0.45 \pm 0.01$ & $96.6 \pm 0.08$ & $5.1 \pm 0.18$ & $2.2 \pm 0.08$ & $0.10 \pm 0.01$ & $2.04 \pm 0.15$ & $97.9 \pm 0.09$ & $6.8 \pm 0.22$ & $2.9 \pm 0.09$ & $-1.3 \pm 0.20$ \\
\hline \multicolumn{11}{|c|}{ W43-MM2 Ridge } \\
\hline W43-MM7 & $0.53 \pm 0.01$ & $95.0 \pm 0.02$ & $5.3 \pm 0.04$ & $2.3 \pm 0.02$ & $0.26 \pm 0.01$ & $1.83 \pm 0.19$ & $93.8 \pm 0.49$ & $12.3 \pm 1.24$ & $5.2 \pm 0.53$ & $1.2 \pm 0.50$ \\
\hline W43-MM17 & $0.60 \pm 0.67$ & $90.6 \pm 0.10$ & $2.5 \pm 0.17$ & $1.1 \pm 0.07$ & $0.21 \pm 0.32$ & $1.21 \pm 0.12$ & $90.9 \pm 0.25$ & $6.8 \pm 0.62$ & $2.9 \pm 0.26$ & $-0.3 \pm 0.27$ \\
\hline W43-MM3 & $0.55 \pm 0.01$ & $93.7 \pm 0.01$ & $4.5 \pm 0.02$ & $1.9 \pm 0.01$ & $0.14 \pm 0.01$ & $3.00 \pm 0.13$ & $93.2 \pm 0.12$ & $6.9 \pm 0.29$ & $2.9 \pm 0.12$ & $0.5 \pm 0.12$ \\
\hline W43-MM2 & $0.78 \pm 0.01$ & $90.7 \pm 0.01$ & $4.5 \pm 0.02$ & $1.9 \pm 0.01$ & $0.11 \pm 0.01$ & $4.25 \pm 0.18$ & $91.0 \pm 0.12$ & $8.0 \pm 0.35$ & $3.4 \pm 0.15$ & $-0.3 \pm 0.12$ \\
\hline W43-MM22 & $0.46 \pm 0.01$ & $88.5 \pm 0.05$ & $4.1 \pm 0.09$ & $1.7 \pm 0.04$ & $0.10 \pm 0.01$ & $1.69 \pm 0.19$ & $88.3 \pm 0.26$ & $6.5 \pm 0.73$ & $2.8 \pm 0.31$ & $0.2 \pm 0.27$ \\
\hline W43-MM2 ridge ${ }^{\mathrm{e}}$ & $0.55 \pm 0.01$ & $90.7 \pm 0.01$ & $4.5 \pm 0.02$ & $1.9 \pm 0.01$ & $0.14 \pm 0.01$ & $1.83 \pm 0.19$ & $91.0 \pm 0.12$ & $6.9 \pm 0.29$ & $2.9 \pm 0.12$ & $-0.3 \pm 0.12$ \\
\hline
\end{tabular}

Notes.

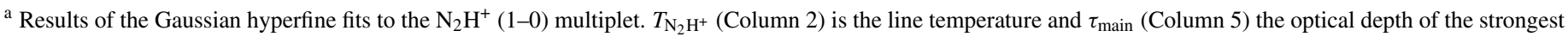
$\mathrm{N}_{2} \mathrm{H}^{+}(1-0)$ multiplet, related by the equation $T_{\mathrm{N}_{2} \mathrm{H}^{+}}=T_{\mathrm{MB}} \times \tau_{\text {main }}$.

b Results of the Gaussian fits to the $\mathrm{SiO}(2-1)$ lines.

c The shift between the peak velocities of the $\mathrm{N}_{2} \mathrm{H}^{+}(1-0)$ and $\mathrm{SiO}(2-1)$ lines is measured as $\Delta_{V_{\mathrm{LSR}}}=V_{\mathrm{LSR}}\left(\mathrm{N}_{2} \mathrm{H}^{+}\right)-V_{\mathrm{LSR}}(\mathrm{SiO})$.

$\mathrm{d}$ The one-dimensional velocity dispersion is calculated as $\sigma_{1 \mathrm{D}}=\left(\Delta V_{\mathrm{FWHM}}\right) /(\sqrt{8 \ln 2})$, using Column 4 for $\mathrm{N}_{2} \mathrm{H}^{+}$and Column 10 for SiO.

e The values given for the ridges are the mean values of all pixels contained in each ridge.

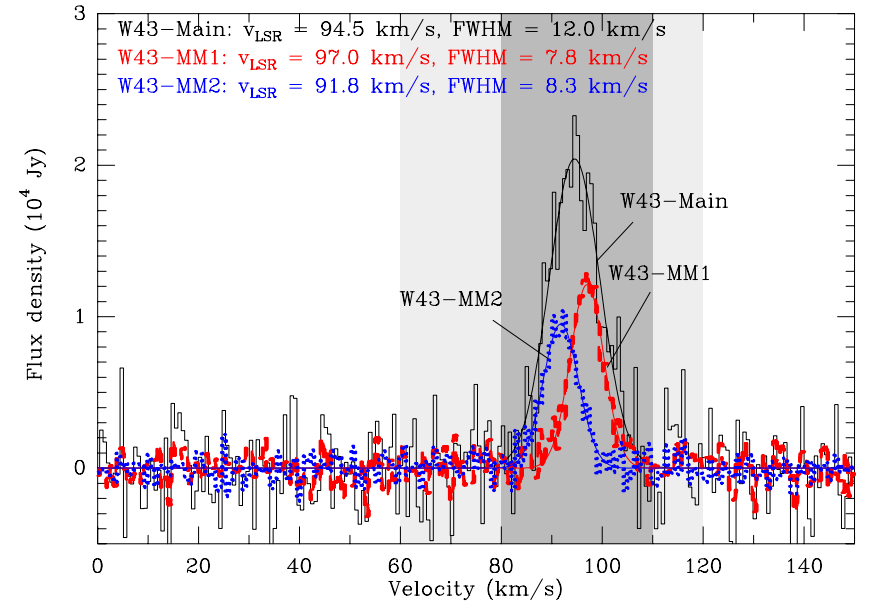

Figure 4. $\mathrm{SiO}(2-1)$ accumulated line spectra resulting from the sum of all spectra observed in the entire W43-Main area (black line), compared to those summed over the W43-MM1 ridge (red dashed line) and W43-MM2 ridge (blue dotted line). The main and complete velocity ranges of W43 defined by Nguyen Luong et al. (2011b) are indicated with light and dark gray filling.

(A color version of this figure is available in the online journal.)

region, as well as the spectra integrated over the W43-MM1 and the W43-MM2 ridges defined in Section 3.1. The spectrum integrated over the entire W43-Main region spans a velocity range of $80-110 \mathrm{~km} \mathrm{~s}^{-1}$, in agreement with the main ${ }^{13} \mathrm{CO}(1-0)$ velocity range of W43 derived in Nguyen Luong et al. (2011b). Note that Carlhoff et al. (2013) used the ${ }^{13} \mathrm{CO}(2-1)$ data of our IRAM large program to refine the main velocity range for the W43 complex to $78-110 \mathrm{~km} \mathrm{~s}^{-1}$. The similarity in velocity ranges between the ${ }^{13} \mathrm{CO}(1-0)$ and $\mathrm{SiO}(2-1)$ lines is remarkable since $\mathrm{SiO}$ lines are generally tracing shocks from outflows and are thus, expected to have very extended wings (e.g., Schilke et al. 1997). Some $\mathrm{SiO}$ emission arising from a collection of outflows driven by protostars forming in the W43-MM1 and W43-MM2 ridges could be hidden in these broad lines. The $\mathrm{SiO}(2-1)$ lines of Figure 4 seem, however, to be dominated by one line component without velocity wings. We fitted Gaussian profiles to all three averaged spectra and obtained $V_{\mathrm{LSR}}$ of $97.0 \mathrm{~km} \mathrm{~s}^{-1}$ for the W43-MM1 ridge, $91.8 \mathrm{~km} \mathrm{~s}^{-1}$ for the W43-MM2 ridge, and $94.5 \mathrm{~km} \mathrm{~s}^{-1}$ for the entire W43-Main. The FWHM line width measured toward the W43-MM1 and W43-MM2 ridges are both $\sim 8 \mathrm{~km} \mathrm{~s}^{-1}$, while that of the averaged spectrum in the entire W43-Main is somewhat larger, $\sim 12 \mathrm{~km} \mathrm{~s}^{-1}$. The integrated peak $\mathrm{SiO}$ fluxes in the W43-MM1 and W43-MM2 ridges are of the same order: $\sim 1.2 \times 10^{4} \mathrm{Jy}$ (see Table 3 ).

The intensity map of the $\mathrm{SiO}(2-1)$ emission, integrated from 80 to $110 \mathrm{~km} \mathrm{~s}^{-1}$, is shown in Figure 5 for the W43Main mini-starburst. The $\mathrm{SiO}$ emission above a $3 \sigma$ level, i.e., $>0.8 \mathrm{~K} \mathrm{~km} \mathrm{~s}^{-1}$, appears as two extended features following the density structure of the two W43-MM1 and W43-MM2 ridges (see and compare the blue $\mathrm{SiO}(2-1)$ and black $N_{\mathrm{H}_{2}}>10^{23} \mathrm{~cm}^{-2}$ contours in Figure 5). A closer inspection shows that there is an exception north of the W43-MM33 position since the $\mathrm{SiO}$ emission peak is offset by $\sim 0.5 \mathrm{pc}\left(\sim 15^{\prime \prime}\right)$ from the dust column density peak. The spatially integrated $\mathrm{SiO}$ fluxes in the W43MM1 and W43-MM2 regions are $\sim 39 \mathrm{~K} \mathrm{~km} \mathrm{~s}^{-1} \mathrm{pc}^{2}$ for areas of $\sim 12$ and $\sim 16 \mathrm{pc}^{2}$, respectively. Their $\mathrm{SiO}$ emitting regions have mean dust column density of $\sim 1.5 \times 10^{23} \mathrm{~cm}^{-2}$ and $\sim 5.9 \times 10^{23} \mathrm{~cm}^{-2}$, respectively. The strongest $\mathrm{SiO}$ emission peaks are at the position of massive dense cores in the W43MM1 and W43-MM2 ridges, especially toward W43-MM1 and W43-MM2, but $\mathrm{SiO}$ emission is also detected toward other more isolated sources. Beuther \& Sridharan (2007) observed five locations in the W43-Main region, among which two remained undetected in our lower-sensitivity image (see Figure 5). Their observations suggest that the $\mathrm{SiO}(2-1)$ emission would be even more extended if observed with a higher sensitivity. 

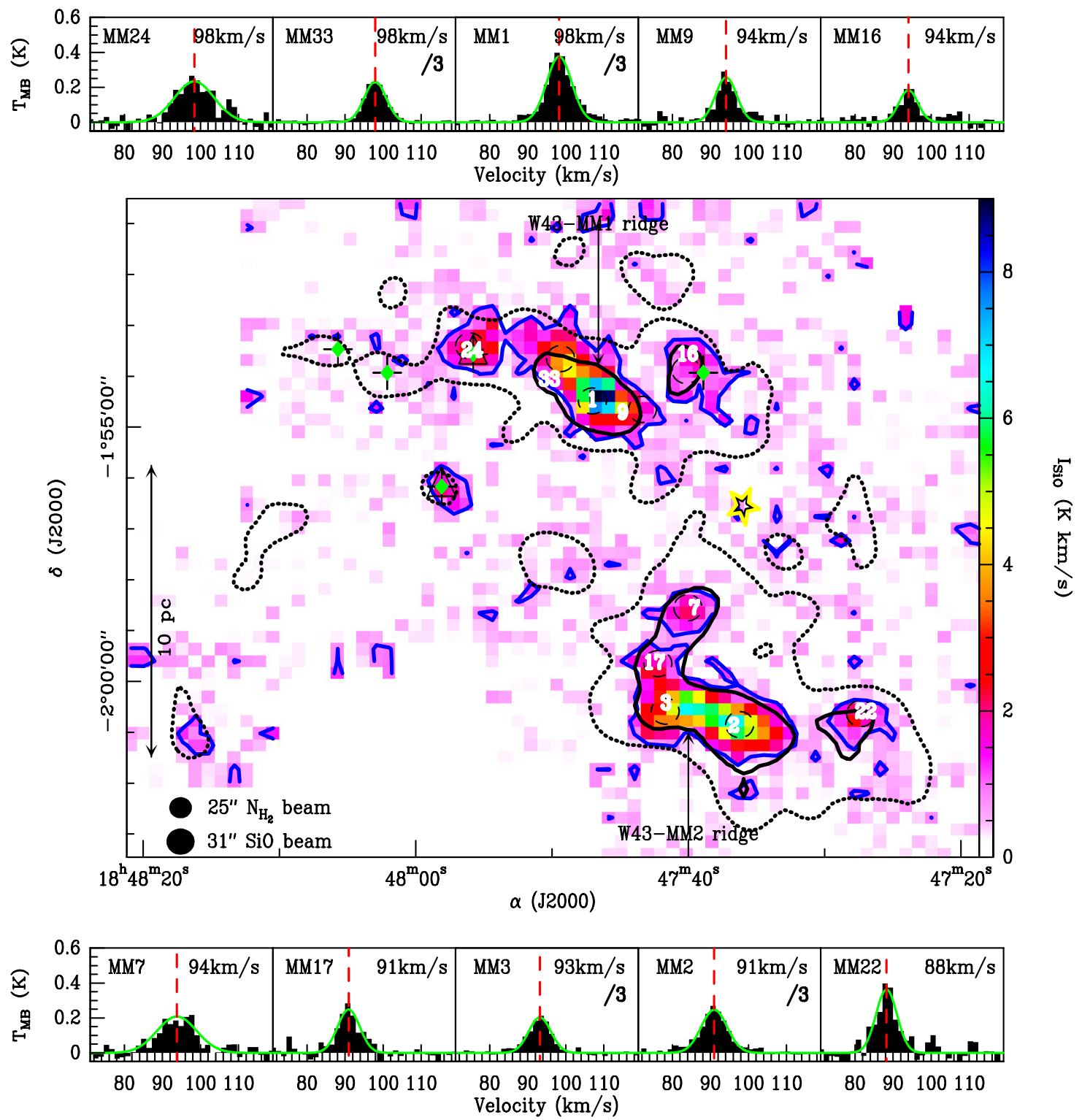

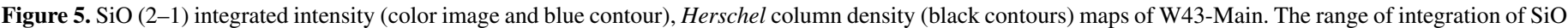

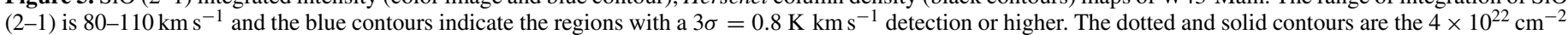

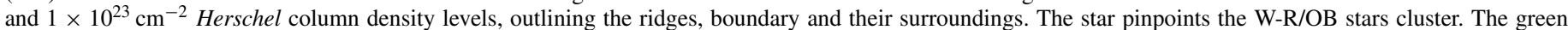

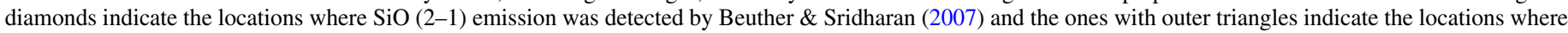

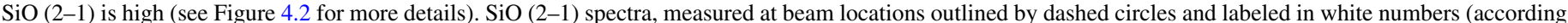

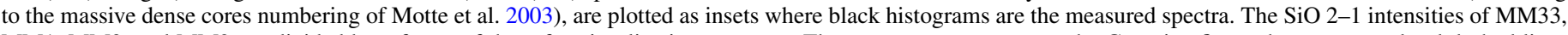

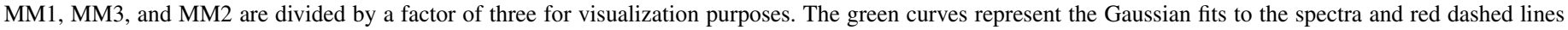
mark the sources peak velocity derived from the fits.

(A color version of this figure is available in the online journal.)

To probe the kinematics of shocked gas traced by $\mathrm{SiO}$ emission in more detail, we plotted the $\mathrm{SiO}$ spectra toward several peak positions in Figure 5. These spectra are gridded at the HPBW of $\sim 31^{\prime \prime}$ or $\sim 0.9 \mathrm{pc}$ at a distance of $6 \mathrm{kpc}$; thus, each box represents a beam-separated independent spectrum. These spectra illustrate that the $\mathrm{SiO}$ emission is resolved and extended over several beams toward both the W43-MM1 and W43-MM2 ridges. The line parameters change from one location to another but most of the $\mathrm{SiO}$ spectra can be very well approximated by a single Gaussian profile (see Table 4). The velocity dispersions of $\mathrm{SiO}(2-1)$ spectra are larger than those of $\mathrm{N}_{2} \mathrm{H}^{+}(1-0)$ spectra: $\sim 2.9 \mathrm{~km} \mathrm{~s}^{-1}$ instead of $\sim 2.2 \mathrm{~km} \mathrm{~s}^{-1}$. W43-MM24 and W43-MM7, both at one end of the ridges, are the locations with the broadest $\mathrm{SiO}(2-1)$ and $\mathrm{N}_{2} \mathrm{H}^{+}$lines (see Table 4) compared to the rest of the ridges. The comparison of the $\mathrm{N}_{2} \mathrm{H}^{+}(1-0)$ and $\mathrm{SiO}(2-1)$ peak velocities reveals a median offset of $\sim 1 \mathrm{~km} \mathrm{~s}^{-1}$ in the W43-MM1 ridge (see Table 4).

\subsection{SiO (2-1) Luminosity and Velocity Extent}

We measured the luminosity and the velocity extent (or full width at zero power, FWZP) of the $\mathrm{SiO}(2-1)$ lines detected in beam-averaged, $\sim 0.9 \mathrm{pc}$, pixels. To do so, we first regridded the $\mathrm{SiO}$ spectra to $\sim 31^{\prime \prime}$ pixels, corresponding to the beam size of our observations and $\sim 0.9 \mathrm{pc}$ at $6 \mathrm{kpc}$ from the Sun. We then selected 17 spectra above the $3 \sigma$ level detection observed in the W43-MM1 ridge and 18 in the W43-MM2 ridge, and thus were located within the blue contour of Figure 5. The velocity extents, luminosities, and intensities of these $\mathrm{SiO}(2-1)$ spectra 

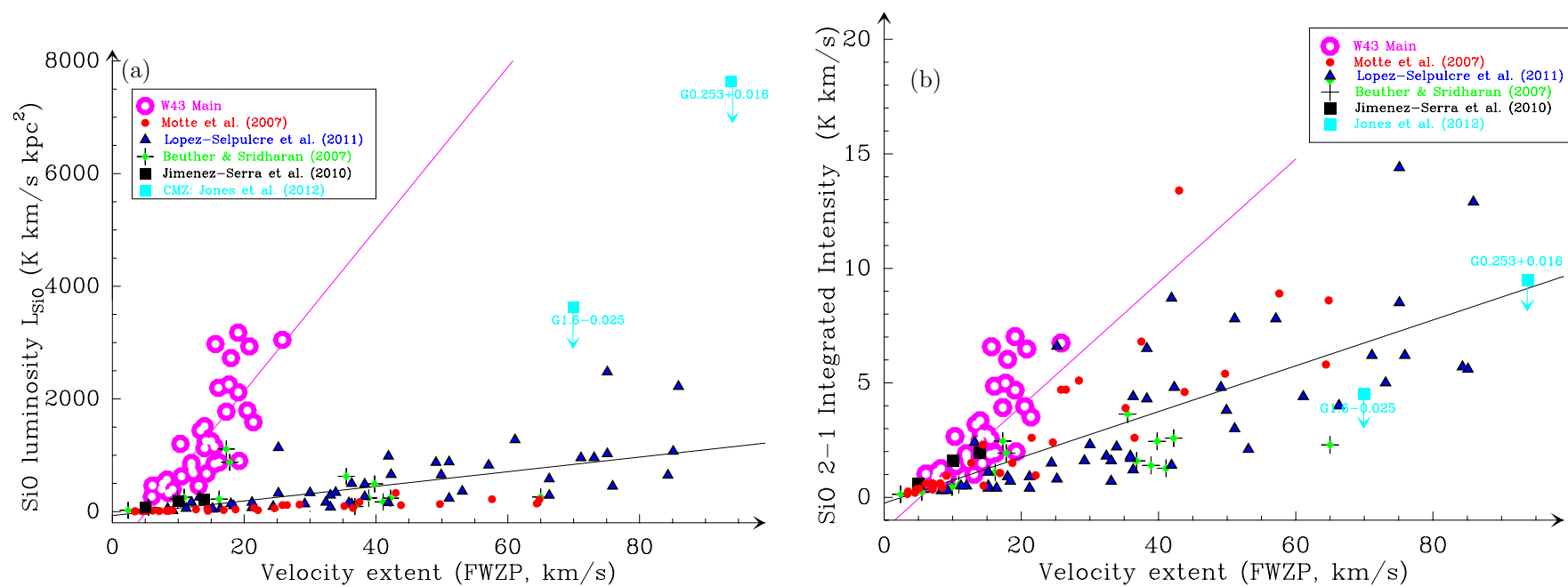

Figure 6. Luminosity (a) and intensity (b) of the SiO lines detected in W43-Main (pink open circles) as a function of their FWZP velocity extent. The 35 circles represent the $\mathrm{SiO}$ measurements of all of the $31^{\prime \prime}$ beam independent points measured above $3 \sigma$ at the IRAM $30 \mathrm{~m}$ telescope. They are compared with those of the $\mathrm{SiO}$ lines measured toward the Cygnus X massive dense cores (red filled circles; Motte et al. 2007), massive star-forming cores (blue filled triangles; López-Sepulcre et al. 2011), and high-mass protostellar objects (green diamonds and plus signs; Beuther \& Sridharan 2007). Most of the latter sources host high-mass protostars, driving powerful SiO outflows. W43-Main and IRDC G035.39-00.33 (black filled squares; Jiménez-Serra et al. 2010) display SiO emission that may be associated with colliding flows shocks. The pink solid line is the linear fit for W43-Main while the black solid line is the one for sources in all other samples taken together (except the CMZ; see Section 4.2). The CMZ sources (blue squares; Jones et al. 2012) have SiO emission whose nature is still not clearly known.

(A color version of this figure is available in the online journal.)

were then compared in Table 5 to the values determined for a few selected samples (López-Sepulcre et al. 2011; Beuther \& Sridharan 2007; Motte et al. 2007; Jiménez-Serra et al. 2010). The velocity extents of the $\mathrm{SiO}$ lines in W43-Main were defined above the $3 \sigma$, i.e., $0.05 \mathrm{~K} \mathrm{~km} \mathrm{~s}^{-1}$ level. This level is eight times less sensitive than G035.39-00.33 and three time less sensitive than the rest of the comparative studies (for details, see Table 5). However, even with higher sensitivity, G035.39-00.33 do not show wings in spectra. Therefore, we expect both of our velocity extents and $\mathrm{SiO}$ intensities to be directly comparable with those reported in the comparative studies within a factor of $\sim 3$ times.

The sources from the López-Sepulcre et al. (2011), Beuther \& Sridharan (2007), Motte et al. (2007), and Jiménez-Serra et al. (2010) samples have all been studied in $\mathrm{SiO}(2-1)$ with the IRAM $30 \mathrm{~m}$ telescope and also with a $\sim 31^{\prime \prime}$ beam. They span a large variety of distances: $\sim 1.4 \mathrm{kpc}$ for the Cygnus $\mathrm{X}$ massive dense cores identified by Motte et al. (2007), 1.7-5.7 kpc for the massive star-forming cores of López-Sepulcre et al. (2011), 0.7-7.3 kpc for high-mass protostellar objects (Beuther \& Sridharan 2007), and $\sim 2.9 \mathrm{kpc}$ for IRDC G035.39-00.33 (Jiménez-Serra et al. 2010). In the first three studies, the SiO (2-1) emission is mainly interpreted as a tracer of protostellar outflows; in the last one, the $\mathrm{SiO}$ emission is interpreted as associated with colliding flows. CO outflows of high-mass protostars are known to typically be $\sim 0.05-0.8 \mathrm{pc}$ long (e.g., Beuther et al. 2002; Duarte-Cabral et al. 2013). The SiO emission in the three comparative studies arises from local regions where high-velocity shocks are developing and thus, should be confined within a $\sim 31^{\prime \prime}$ beam for these $\sim 2.9 \mathrm{kpc}$ sources.

None of the published $\mathrm{SiO}$ measurements of the $\mathrm{CMZ}$ were observed with the same transition and spatial resolution as in our W43 observations. To compare our W43 SiO observations with this well-known region, we used the $\mathrm{SiO}(2-1)$ measurements of the G0.253+0.016 and G1.6-0.025 sources of the CMZ obtained with the MOPRA telescope (Jones et al. 2012). The larger beams $\left(40^{\prime \prime}\right)$ of this observation overestimate their $\mathrm{SiO}(2-1)$ in- tegrated intensity and luminosity compared to ours but probably not by more than $50 \%$. A distance of $8 \mathrm{kpc}$ is assumed. The origin of the $\mathrm{SiO}$ emission from the $\mathrm{CMZ}$ clouds is still a matter of debate since star formation may be inhibited (Menten et al. 2009; Kruijssen et al. 2013). For W43-Main and the outflow sources of the comparative samples, we estimated the $\mathrm{SiO}(2-1)$ luminosity, $L_{\mathrm{SiO}(2-1)}$, from the integrated intensity, $\int T_{\mathrm{MB}} d v$, via:

$$
\begin{aligned}
L_{\mathrm{SiO}(2-1)} & =4 \pi \times d^{2} \times \int T_{\mathrm{MB}} d v \\
& \simeq 450 \mathrm{~K} \mathrm{~km} \mathrm{~s}^{-1} \mathrm{kpc}^{2} \times\left(\frac{d}{6 \mathrm{kpc}}\right)^{2} \frac{\int T_{\mathrm{MB}} d v}{1 \mathrm{~K} \mathrm{~km} \mathrm{~s}^{-1}} \\
& \simeq 1.9 \times 10^{-5} L_{\odot} \times\left(\frac{d}{6 \mathrm{kpc}}\right)^{2} \frac{\int T_{\mathrm{MB}} d v}{1 \mathrm{~K} \mathrm{~km} \mathrm{~s}^{-1}} .
\end{aligned}
$$

The conversion of the $\mathrm{SiO} 2-1$ luminosity unit from $\mathrm{K} \mathrm{km} \mathrm{s}^{-1} \mathrm{kpc}^{2}$ to $L_{\odot}$ is discussed in Appendix C. Luminosity allows a more direct comparison of published intensities with those measured in W43-Main. For sources closer than W43, Equation (6) is a proxy of the dilution of their intensities measured within $\sim 0.1-0.9 \mathrm{pc}$ beams to the largest physical scale, here $0.9 \mathrm{pc}$. This is correct for the unresolved $\mathrm{SiO}$ emission of outflow sources (e.g., López-Sepulcre et al. 2011; Beuther \& Sridharan 2007; Motte et al. 2007), but it is obviously incorrect for IRDC G035.39-00.33 whose emission is extended (Jiménez-Serra et al. 2010). The comparison of intensities rather than luminosities is more appropriate in such cases but with an implicit assumption that the sources have a constant intensity emission over at least $0.9 \mathrm{pc}$.

Figure 6 shows the relations for both the $\mathrm{SiO}(2-1)$ luminosity (panel (a)) and integrated intensity (panel (b)) as a function of their velocity extent. We labeled them hereafter as the $L-F W Z P$ and $I_{\text {int }}-F W Z P$ relations. We do not include the measurements of the CMZ in the fits since their $L$-FWZP strays from the W43 sample and the comparative samples. The most notable trait is that the $\mathrm{SiO}$ emission in W43-Main does not spread over more than 


\begin{tabular}{|c|c|c|c|c|c|c|c|c|c|}
\hline \multirow{2}{*}{$\begin{array}{l}\text { Source Samples }^{\mathrm{a}} \\
\text { (1) }\end{array}$} & \multirow{2}{*}{$\begin{array}{c}\langle d\rangle \\
(\mathrm{kpc}) \\
(2)\end{array}$} & \multirow{2}{*}{$\begin{array}{c}3 \sigma \mathrm{rms} \\
\left(\mathrm{K} / \mathrm{km} \mathrm{s}^{-1}\right) \\
(3)\end{array}$} & \multirow{2}{*}{$\begin{array}{c}\langle\mathrm{FWZP}\rangle \\
\left(\mathrm{km} \mathrm{s}^{-1}\right) \\
(4)\end{array}$} & \multirow{2}{*}{$\begin{array}{c}\left\langle L_{\mathrm{SiO}(2-1)}\right\rangle \\
\left(\mathrm{K} \mathrm{km} \mathrm{s}^{-1} \mathrm{kpc}^{2}\right) \\
\text { (5) }\end{array}$} & \multirow{2}{*}{$\begin{array}{c}\left\langle I_{\mathrm{SiO}(2-1)}^{\text {int }}\right\rangle \\
\left(\mathrm{K} \mathrm{km} \mathrm{s}^{-1}\right) \\
(6)\end{array}$} & \multicolumn{2}{|c|}{$L=\alpha_{\mathrm{L}} \times \mathrm{FWZP}-\beta_{\mathrm{L}}$} & \multicolumn{2}{|c|}{$I=\alpha_{\mathrm{I}} \times \mathrm{FWZP}-\beta$} \\
\hline & & & & & & $\begin{array}{c}\alpha_{\mathrm{L}}^{\mathrm{b}} \\
\text { (7) }\end{array}$ & $\begin{array}{c}\beta_{\mathrm{L}}^{\mathrm{b}} \\
(8)\end{array}$ & $\begin{array}{l}\alpha_{\mathrm{I}}^{\mathrm{b}} \\
(9)\end{array}$ & $\begin{array}{l}\beta_{\mathrm{I}}^{\mathrm{b}} \\
(10)\end{array}$ \\
\hline W43-Main $(>3 \sigma)$ & 6.0 & 0.05 & 14.2 & 960 & 2.1 & 144 & 751 & 0.27 & 1.42 \\
\hline W43-MM1, 17 beams & 6.0 & 0.05 & 12.3 & 770 & 1.7 & $\ldots$ & $\ldots$ & $\ldots$ & $\ldots$ \\
\hline W43-MM2, 18 beams & 6.0 & 0.05 & 14.6 & 970 & 2.2 & $\ldots$ & $\ldots$ & $\ldots$ & $\ldots$ \\
\hline All comparative studies & 2.9 & & 31.8 & 331 & 3.0 & 13 & 72 & 0.1 & 0.26 \\
\hline M07, 31 sources in Cygnus X & 1.4 & 0.03 & 18.7 & 37 & 1.5 & $\ldots$ & $\ldots$ & $\ldots$ & $\ldots$ \\
\hline LS11, 49 sources & 3.0 & 0.03 & 35.9 & 290 & 2.2 & $\ldots$ & $\ldots$ & $\ldots$ & $\ldots$ \\
\hline BS07, 14 sources & 3.9 & 0.02 & 27.0 & 240 & 1.5 & $\ldots$ & $\ldots$ & $\ldots$ & $\ldots$ \\
\hline $\mathrm{J} 10,3$ beams in $\mathrm{G} 035.39-00.33$ & 2.9 & 0.006 & 10.0 & 330 & 1.6 & $\ldots$ & $\ldots$ & $\ldots$ & $\ldots$ \\
\hline J12, CMZ G0.253+0.016 & 8.0 & 0.006 & 70.0 & 608 & 9.5 & $\ldots$ & $\ldots$ & $\ldots$ & $\ldots$ \\
\hline J12, CMZ G1.6-0.025 & 8.0 & 0.006 & 93.8 & 288 & 4.5 & $\ldots$ & $\ldots$ & $\ldots$ & $\cdots$ \\
\hline
\end{tabular}

Notes.

${ }^{\text {a }}$ References. M07 = Motte et al. (2007); LS11 = López-Sepulcre et al. (2011); BS07 = Beuther \& Sridharan (2007); J10 = Jiménez-Serra et al. (2010), J12 = Jones et al. (2012). Sources types: MDCs = massive dense cores; MSFCs = star-forming cores; HMPOs = high-mass protostellar objects; $\mathrm{CMZ}=$ Central Molecular Zone.

${ }^{\mathrm{b}}$ Linear fit parameters measured from Figures 6(a)-(b). The units of $\alpha_{\mathrm{L}}$ and $\alpha_{\mathrm{I}}$ are $\mathrm{K} \mathrm{kpc}{ }^{2}$ and $\mathrm{K}$ while those of $\beta_{\mathrm{L}}$ and $\beta_{\mathrm{I}}$ are $\mathrm{K} \mathrm{km} \mathrm{s}{ }^{-1} \mathrm{kpc}^{2}$ and K km s${ }^{-1}$. We note that the measurements of CMZ are not included in the fit. 
$25 \mathrm{~km} \mathrm{~s}^{-1}$, in contrast to the high-mass molecular outflows which spread up to $\sim 50 \mathrm{~km} \mathrm{~s}^{-1}$ (e.g., López-Sepulcre et al. 2011). The median velocity extent of $W 43$ is much smaller than the median values in other samples: $\sim 14 \mathrm{~km} \mathrm{~s}^{-1}$ compared to $\sim 32 \mathrm{~km} \mathrm{~s}^{-1}$ (see Table 5). Furthermore, the SiO (2-1) luminosity and intensity of W43-Main pixels are much steeper functions of their velocity extent than the relations measured for other samples (see Figures 6(a)-(b)). The parameters of the best linear fits are given in Table 5. In more detail, with a linear relation between the $\mathrm{SiO}(2-1)$ luminosity and velocity extent such as

$$
L_{\mathrm{SiO}(2-1)}=\alpha_{\mathrm{L}} \times \mathrm{FWZP}_{\mathrm{SiO}(2-1)}-\beta_{\mathrm{L}},
$$

we measured $\alpha_{\mathrm{L}} \sim 140 \mathrm{~K} \mathrm{kpc}^{2}$ for W43-Main and $\alpha_{\mathrm{L}} \sim$ $13 \mathrm{~K} \mathrm{kpc}^{2}$ for the comparative samples taken together. With a similarly linear relation between the $\mathrm{SiO}(2-1)$ integrated intensity and velocity extent, $I_{\mathrm{SiO}(2-1)}=\alpha_{\mathrm{I}} \times \mathrm{FWZP}_{\mathrm{SiO}(2-1)}-\beta_{\mathrm{I}}$, the slope of the W43-Main region remains three times steeper than for the comparative samples (see Table 5).

Despite a large range of distances, the $\mathrm{SiO}(2-1)$ integrated intensities in all sources of the different samples, including W43Main except the CMZ sources, are almost similar: $\left\langle I_{\mathrm{SiO}(2-1)}^{\mathrm{int}}\right\rangle \sim$ $2-3 \mathrm{~K} \mathrm{~km} \mathrm{~s}^{-1}$. In contrast, the median $\mathrm{SiO}(2-1)$ luminosity in W43-Main, $\left\langle L_{\mathrm{SiO}(2-1)}\right\rangle \sim 10^{3} \mathrm{~K} \mathrm{~km} \mathrm{~s}^{-1} \mathrm{kpc}^{2}$, is three times larger than the median value observed for comparative sources. The total SiO (2-1) luminosity measured toward the W43-Main region is $L_{\mathrm{SiO}(2-1)}^{\text {total }} \sim 4 \times 10^{4} \mathrm{~K} \mathrm{~km} \mathrm{~s}{ }^{-1} \mathrm{kpc}^{2}$, with $50 \%$ concentrated in the W43-MM1 and another $50 \%$ concentrated in W43-MM2 ridge surroundings (see Table 3).

Within the three catalogs of massive young stellar objects, we found three sources that lie near the steep fit of the $L-F W Z P$ relation: G34.26+0.15 in the López-Sepulcre et al. (2011) sample and a couple of sources from Beuther \& Sridharan (2007; see Figure 6(a)). The G34.26+0.15 source is an ultra compact $\mathrm{H}$ II region with a strong hot core $\mathrm{SiO}$ emission (Watt \& Mundy 1999), and the two Beuther \& Sridharan (2007) sources in fact correspond to W43-MM24 and another location in W43Main. The SiO line emission observed toward the CMZ also had higher luminosities than the $\mathrm{SiO}$ lines from outflows with the same FWZP (see Figure 6(a)). If the lack of star formation activity in these regions is confirmed (see Section 5.1), other mechanisms such as violent cloud-cloud collisions could be responsible for producing $\mathrm{SiO}$ in the CMZ (Martin-Pintado et al. 1997; Requena-Torres et al. 2006).

\subsection{SiO Column Density}

Assuming that the $\mathrm{SiO}$ emission is optically thin in shocked regions $(\tau \ll 1)$ and that the local thermal equilibrium (LTE) hypothesis is valid, we can, in principle, calculate the $\mathrm{SiO}$ column density from the integrated intensity by solving the basic radiative transfer equation (Rohlfs \& Wilson 2000). The total $\mathrm{SiO}(2-1)$ column density can be estimated with the following equation:

$$
\begin{aligned}
N_{\mathrm{tot}}^{\mathrm{SiO}(2-1)}= & \frac{3 k}{8 \pi^{3} S \mu_{s}^{2} v} \frac{Q_{\mathrm{rot}}\left(T_{\mathrm{ex}}\right)}{g_{u} g_{K} g_{s}} \exp \left(\frac{E_{u}}{k T_{\mathrm{ex}}}\right) \int \frac{T_{\mathrm{MB}}}{f} d V \\
\simeq & 1.98 \times 10^{10} \mathrm{~cm}^{-2} \times \frac{1}{f} \times Q_{\mathrm{rot}}\left(T_{\mathrm{ex}}\right) \\
& \times \exp \left[0.0625\left(\frac{T_{\mathrm{ex}}}{100 \mathrm{~K}}\right)^{-1}\right] \times \frac{\int T_{\mathrm{MB}} d V}{1 \mathrm{~K} \mathrm{~km} \mathrm{~s}^{-1}},
\end{aligned}
$$

where $k$ is the Boltzmann constant and $v=86.846 \mathrm{GHz}$ is the emitting frequency. The line strength $(S=2.0)$, the dipole moment $\left(\mu_{s}=3.098 \mathrm{D}\right)$, and the upper level energy $\left(\frac{E_{u}}{k}=6.25 \mathrm{~K}\right)$ of the $\mathrm{SiO}(2-1)$ transition were taken from the splatalogue catalog. $Q_{\text {rot }}\left(T_{\text {ex }}\right)$ is the partition function which was extrapolated, for a particular excitation temperature, from the tabulated values of the Cologne Database for Molecular Spectroscopy $^{24}$ (CDMS; Müller et al. 2005). The rotational degeneracy is given by $g_{u}=2 J_{u}+1=5$, while the $\mathrm{K}$ and nuclear spin degeneracies are $g_{K}=1$ and $g_{s}=1$. A beam filling factor of $f=1$ is justified in our study since the $\mathrm{SiO}$ emission is extended in W43-Main and fills the entire beam as confirmed by interferometric observations (F. Louvet et al., in preparation). We recall that the LTE calculation relies on the assumption that the gas density is larger than the critical density of the $\mathrm{SiO}(2-1)$ transition given by

$$
n_{\mathrm{crit}}=\frac{A_{\mathrm{ul}}}{\sigma \sqrt{\frac{3 k T_{\mathrm{ex}}}{m_{\mathrm{H}}}}} \simeq 2.93 \times 10^{5} \mathrm{~cm}^{-3}\left(\frac{T_{\mathrm{ex}}}{100 \mathrm{~K}}\right)^{-1 / 2},
$$

where the Einstein coefficient $\left(A_{\mathrm{ul}}=2.93 \times 10^{-5} \mathrm{~s}^{-1}\right)$ and the cross-section $\left(\sigma=10^{-15} \mathrm{~cm}^{-2}\right)$ of the $\mathrm{SiO}$ molecule are taken from the splatalogue catalog. $\sqrt{3 k T_{\mathrm{ex}} / m_{\mathrm{H}}}$ is the approximate ensemble average velocity of the collision partner, hydrogen in this case. Equation (8) yields a critical density of $n_{\text {crit }}=0.3-65 \times 10^{4} \mathrm{~cm}^{-3}$ for an excitation temperature ranging from $10^{6} \mathrm{~K}$ to $20 \mathrm{~K}$. The median density values of the W43-MM1 and W43-MM2 ridges, $n_{\mathrm{H}_{2}} \sim 6.0 \times 10^{4} \mathrm{~cm}^{-3}$ and $3.4 \times 10^{4} \mathrm{~cm}^{-3}$ (see Table 3 ), prove that there are at least some parts of these cloud ridges where the density surpasses the critical density. Therefore, Equation (8) can be used as a good upper limit estimation of the $\mathrm{SiO}$ column density. If the $\mathrm{SiO}$ emission is optically thick, the $\mathrm{SiO}$ column density must be increased by a factor of $\sim \tau /(1-\exp (-\tau))$.

Assuming conservative shock temperatures, $T_{\mathrm{ex}}=20-10^{6} \mathrm{~K}$ in Equation (8), we derived the $\mathrm{SiO}(2-1)$ column density of each of the W43-Main points of Figure 6. We varied the excitation temperatures from a minimum of $T_{\mathrm{ex}}=T_{\text {dust }}=20 \mathrm{~K}$ to a maximum $T_{\mathrm{ex}}=10^{6} \mathrm{~K}$, which is a typical temperature range for shocks (Draine 1980; Chièze et al. 1998). As shown in Figures 1(a) and 5(a) of Lesaffre et al. (2013), each maximum gas temperature can be related to a single shock velocity for a specific set of inputs. Therefore, the observed column density plotted in Figure 7(a) is almost directly comparable to the theoretical column density plotted in Figure 7(b) (see Section 5.2 for a fuller discussion of the models). For instance, a C-type shock with a low velocity of $\sim 7.5 \mathrm{~km} \mathrm{~s}^{-1}$ can easily reach a temperature of $\sim 3000 \mathrm{~K}$ (see Lesaffre et al. 2013 and Section 5.2). Depending on the model, the $\mathrm{SiO}$ molecules could be formed either in the shock front or just after it in the postshock gas, thus arguing for excitation temperatures from a few thousand to a few hundred Kelvin. This is coherent with the fact that at $\sim 1900 \mathrm{~K}, \mathrm{Si}$ starts to evaporate from ice mantles (e.g., Gusdorf et al. 2008b). The SiO column density of the 35 independent $0.9 \mathrm{pc}$ beam points, detected above our $3 \sigma$ level in the W43-MM1 and W43-MM2 ridges plus surroundings, are shown in Figure 7(a) as a function of $T_{\mathrm{ex}}$. Our $\mathrm{SiO}$ column density thus ranges from $5 \times 10^{11} \mathrm{~cm}^{-2}$ to $10^{16} \mathrm{~cm}^{-2}$, with a mean value of $\sim 6 \times 10^{13} \mathrm{~cm}^{-2}$. Such values are typical for shocked regions associated with the most powerful outflows

\footnotetext{
24 The CMDS catalog can be searched here: http://www.astro.uni-koeln.de/cdms
} 

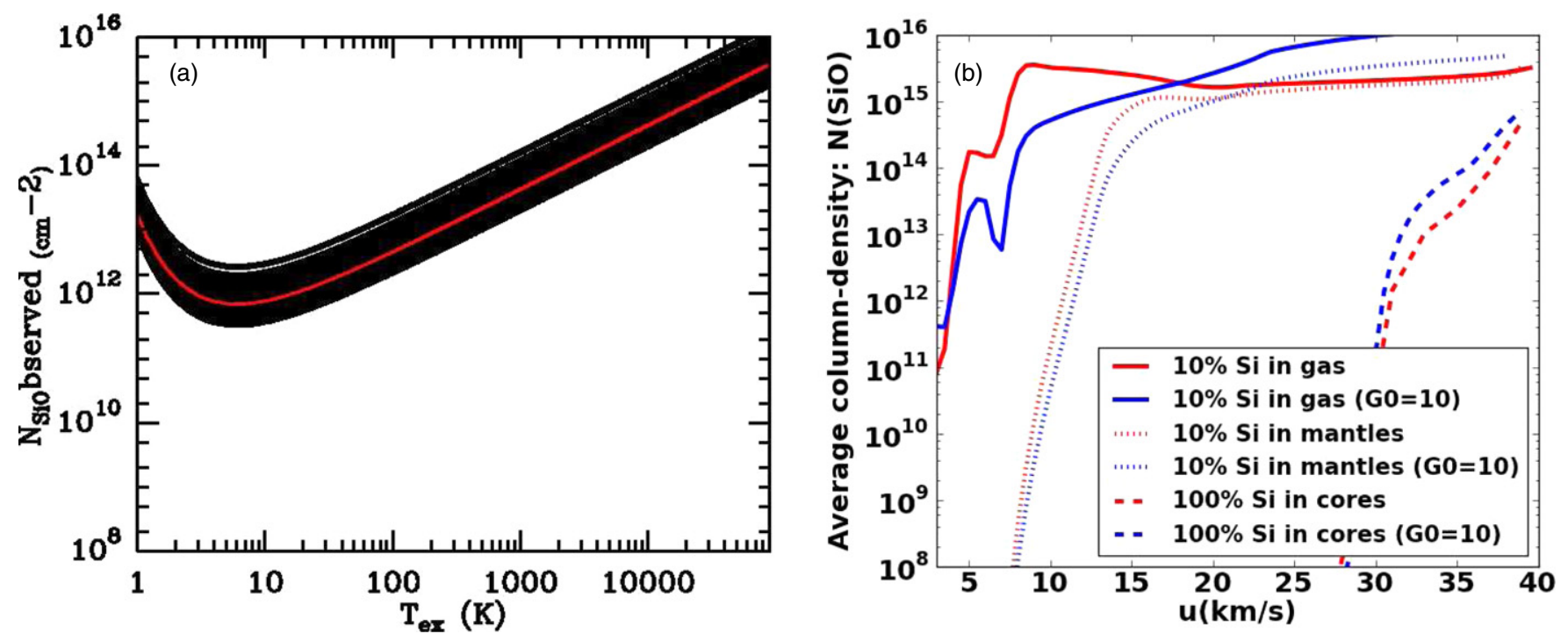

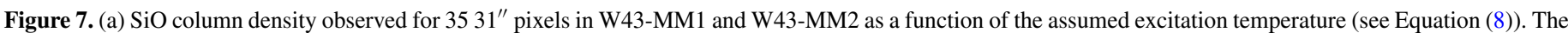

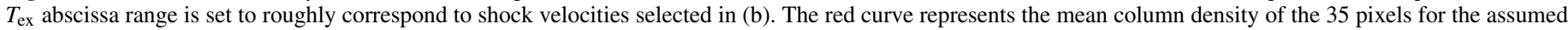

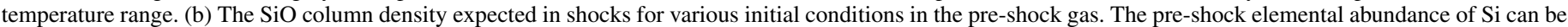

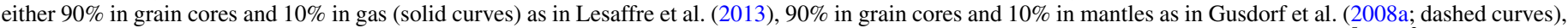

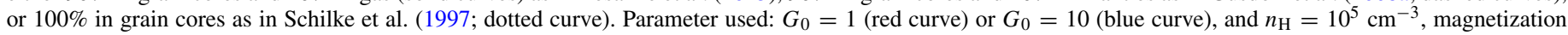
parameter $b=3$ (see Lesaffre et al. 2013 for a more detailed discussion on the model and the input parameters).

(A color version of this figure is available in the online journal.)

driven by high-mass star-forming regions (e.g., G5.89-0.39 and G34.26 by Acord et al. 1997; Hatchell et al. 2001) or observed toward the CMZ (Yusef-Zadeh et al. 2013).

\section{DISCUSSION}

We discuss the origin of the $\mathrm{SiO}$ emission in Sections 5.1 and 5.2 and their implications on the formation of ridges and the subsequent star formation processes in Section 5.3.

\subsection{Observational Constraints on the Origin of the $\mathrm{SiO}$ Emission in the W43-MM1 and W43-MM2 Ridges}

Here, we review the various physical processes that could explain the bright $\mathrm{SiO}(2-1)$ emission spreading along the W43-MM1 and W43-MM2 ridges. As will be explained in Section 5.2, all $\mathrm{SiO}$ emission stem from shocks. We investigate the hypotheses of high-velocity shocks $\left(v_{\text {shock }} \sim\right.$ $20-50 \mathrm{~km} \mathrm{~s}^{-1}$ ) in outflows, moderate- to high-velocity shocks $\left(v_{\text {shock }} \sim 10-20 \mathrm{~km} \mathrm{~s}^{-1}\right)$ in PDRs and hot cores, and lowvelocity shocks $\left(v_{\text {shock }} \leqslant 10 \mathrm{~km} \mathrm{~s}^{-1}\right)$ associated with cloud collision.

$\mathrm{SiO}$ emission coming from outflows driven by young stellar objects is usually caused by high-velocity shocks $\left(v_{\text {shock }} \sim\right.$ $20-50 \mathrm{~km} \mathrm{~s}^{-1}$; Schilke et al. 1997). This mechanism, which should translate into high-velocity spectral wings, could play a role in W43-Main since it is an active star-forming region. However, the SiO lines observed in W43-Main do not spread over more than $25 \mathrm{~km} \mathrm{~s}^{-1}$, in contrast with lines measured toward high-mass protostellar outflows which have up to $\sim 50$ $\mathrm{km} \mathrm{s}^{-1}$ wings (e.g., López-Sepulcre et al. 2011; Motte et al. 2007; see Figures 6(a)-(b)). As it will be shown in F. Louvet et al. (in preparation), from a higher-angular resolution $\mathrm{SiO}$ imaging, beam dilution and sensitivity limit may partly explain why the protostellar outflows of the W43-MM1, W43-MM2, and W43-MM3 massive dense cores are hardly seen. We cannot, however, rule out the scenario of a widespread population of low-mass protostars driving outflows along the W43-MM1 and W43-MM2 ridges. Higher-angular resolution and higher sensitivity $\mathrm{SiO}$ line and dust continuum mappings are necessary to confirm/denounce it (see F. Louvet et al., in preparation)

$\mathrm{SiO}$ emission tracing irradiated shocks in PDRs have been observed to be associated with medium-velocity shocked gas $\left(v_{\text {shock }} \sim 10-20 \mathrm{~km} \mathrm{~s}^{-1}\right.$; e.g., Schilke et al. 2001). Irradiated shocks are to be expected in the W43-Main mini-starburst region since the cloud is associated with a very luminous cluster $\left(\sim 3.5 \times 10^{6} L_{\odot}\right.$; Smith et al. 1978; Bik et al. 2005). Fast winds and ionization from the OB/W-R stars are observed to be interacting with intermediate-density clouds, which are swept up and accumulated at the periphery of the giant $\mathrm{H}_{\text {II }}$ region (Motte et al. 2003). However, we do not detect SiO emission over the PDR ring (see Figure 5), delineated in near-infrared and centimeter continuum emission (Motte et al. 2003; Carlhoff et al. 2013). Moreover, the process of medium-velocity shocks in irradiated PDRs is rather unlikely to develop in the higherdensity and thus better shielded cloud structures that are the W43-MM1 and W43-MM2 ridges. We prove this statement by making shock models for the solar or stronger radiation fields, i.e., $G_{0}=1$ and $G_{0}=10$. The $\mathrm{SiO}$ production is slightly more efficient in the case of $v_{\text {shocks }}>15 \mathrm{~km} \mathrm{~s}^{-1}$ with $G_{0}=10$ but the weaker radiation field seems to produce more $\mathrm{SiO}$ for low-velocity shocks (see Figure 7(b)).

We cannot neglect the impact of the OB cluster on the ridges via its radiation pressure and UV photons. However, as shown by Krumholz \& Matzner (2009), even for the most powerful clusters in the Galaxy, the radiation pressure effect is stalled at a radius much smaller than $5 \mathrm{pc}$. Moreover, the ridges are very dense regions with $n_{\mathrm{H}_{2}}>10^{4} \mathrm{~cm}^{-3}$, the penetrating depths of UV photons from a cluster with $G_{0} \sim 10^{5}$, similar to the W43 OB cluster, will decrease rapidly and reach a value of $G_{0}<10$ within the ridge (Meixner \& Tielens 1993). Therefore, the W43 OB cluster cannot be the main cause of the extended 
shocks observed in the W43 ridges, although it could still contribute.

Hot cores and hot corinos are emitting $\mathrm{SiO}$ lines that trace high-velocity $\left(v_{\text {shock }} \sim 50 \mathrm{~km} \mathrm{~s}^{-1}\right.$; Hatchell et al. 2001; Jørgensen et al. 2011) shocks associated with the strong thermal heating of young stars. The $\mathrm{SiO}$ lines with hot core origins could account for part of the emission observed toward the high-mass protostars of W43-Main. However, similar to the outflow hypothesis, our analysis of $\mathrm{SiO}$ lines did not reveal many high-velocity components (see Figures 4 and 6(a)-(b)) and the protostellar content of the W43-MM1 and W43-MM2 ridges must still be assessed. This scenario is thus not very likely.

Therefore, none of the physical processes usually advocated to explain $\mathrm{SiO}$ emission in star-forming regions, i.e., highvelocity or irradiated shocks, reasonably explain all $\mathrm{SiO}(2-1)$ emission observed in W43-Main. Given that the observed SiO lines have Gaussian-like shapes without any high-velocity components (see, e.g., Figure 5), a series of low-velocity, i.e., $v_{\text {shock }} \leqslant 10 \mathrm{~km} \mathrm{~s}^{-1}$, shocks are more appropriate (see Section 5.2). The similar line shapes, in terms of intensity and width, over the two $\sim 10 \mathrm{pc}^{2}$ areas (see Table 4) moreover support the hypothesis of a large-scale mechanism homogeneously producing shocks throughout both the W43-MM1 and W43-MM2 ridges. The similar and overlapping extended morphologies between the shocked gas and the dense gas of ridge structures (see Figures 3 and 5), suggest that the formation of ridges is associated with such shocks. The mechanism at the origin of $\mathrm{SiO}$ emission in W43-Main could thus be colliding flows or a merging of filaments that are interpreted as the processes necessary to build up cloud ridges (e.g., Schneider et al. 2010b; Hennemann et al. 2012).

Jiménez-Serra et al. (2010) also proposed that the $\mathrm{SiO}$ emission observed along IRDC G035.39-00.33 could partly arise from the collision of gas streams that form the higherdensity IRDC structure. Their measurements, however, suffer from a lack of sensitivity and spatial resolution: only three independent beams are above their $3 \sigma$ detection level. A detailed comparison shows that the $\mathrm{SiO}$ (2-1) observed along each of the W43-MM1 and W43-MM2 ridges are $>30$ times more intense and $\sim 10$ times more extended than that of IRDC G035.39-00.33. In Figure 5, we detected SiO (2-1) integrated intensities of $1-11 \mathrm{~K} \mathrm{~km} \mathrm{~s}^{-1}$ over $\sim 12 \mathrm{pc}^{2}$ and $\sim 16 \mathrm{pc}^{2}$ areas along the W43-MM1 and W43-MM2 ridges and their immediate surroundings, respectively. These values are to be compared with the $0.02-0.05 \mathrm{~K} \mathrm{~km} \mathrm{~s}^{-1}$ emission observed along the $\sim 1 \mathrm{pc}^{2}$ IRDC G035.39-00.33 filament (Jiménez-Serra et al. 2010; Henshaw et al. 2013; see also Figure 6(b)). The W43-MM1 and W43-MM2 ridges could be two more regions, as IRDC G035.39-00.33, where extended $\mathrm{SiO}$ emission is detected and interpreted as arising from lowvelocity shocks associated with the formation of high-density cloud structures. From the analysis of Section 4.2, we propose to use a linear relation in the luminosity versus velocity extent diagram of Figure 6 (a) to distinguish the low-velocity $(\leqslant 10$ $\left.\mathrm{km} \mathrm{s}^{-1}\right)$ shocks with steep slopes $\left(\alpha_{\mathrm{L}} \simeq 140 \mathrm{~K} \mathrm{kpc}^{2}\right.$ defined in Equation (7)), possibly associated with cloud collision from more classical high-velocity $\left(\sim 20-100 \mathrm{~km} \mathrm{~s}^{-1}\right)$ shocks with flatter slopes $\left(\alpha_{\mathrm{L}}=13 \pm 7 \mathrm{~K} \mathrm{kpc}^{2}\right)$. The $\mathrm{SiO}$ emission in $\mathrm{W} 43$ is as luminous as the $\mathrm{SiO}$ emission associated with extremely high-velocity outflows, despite having narrower velocity range. This may suggest that the low-velocity shocks in W43 are highly efficient in forming/releasing $\mathrm{SiO}$ over an extended region.
Widespread $\mathrm{SiO}$ emission, with velocity dispersion exceeding those observed toward W43-Main, has been reported toward the entire CMZ of our Galaxy (CMZ covering $\sim 10^{4} \mathrm{pc}^{2}$; MartinPintado et al. 1997; Jones et al. 2012). The CMZ is, in general, characterized by much higher temperatures $\left(T_{\text {dust }} \geqslant 50 \mathrm{~K}\right)$ and more turbulence and shears, resulting in larger velocity dispersion $\left(\sigma \geqslant 10-30 \mathrm{~km} \mathrm{~s}^{-1}\right.$; see the recent review by Güsten $\&$ Philipp 2004). Two CMZ clouds with strong and extended $\mathrm{SiO}$ emission, G1.6-0.025 and G0.253+0.016, have been observed to be devoid of signs of massive star formation (Menten et al. 2009; Kauffmann et al. 2013; Kruijssen et al. 2013), however, see Rodríguez \& Zapata (2013). Recent studies by YusefZadeh et al. (2013) still interpreted the very broad SiO lines observed close to Sgr $\mathrm{A}^{*}$ as arising from highly embedded protostellar outflows. We propose that the process explaining the $\mathrm{SiO}$ emission measured in the $\mathrm{CMZ}$ is partly, as in the case of W43-Main, velocity shears arising from cloud collision. Given the much higher shock velocities in CMZ ( $v_{\text {shock }}$ is up to $80 \mathrm{~km} \mathrm{~s}^{-1}$ ) and interstellar radiation field, its $\mathrm{SiO}$ emission could be explained either in the framework of highly irradiated and highly magnetized shock models, or in that of fast J-type shock models (see Section 5.2 for a short review on shock models).

This is in marked contrast with the strong $\mathrm{SiO}$ emission of W43-Main detected at the ridges' standard velocities at rest. In $\mathrm{W} 43$, the $2-5 \mathrm{~km} \mathrm{~s}^{-1}$ velocity dispersion observed for $\mathrm{SiO}(2-1)$ lines and the $<5 \mathrm{~km} \mathrm{~s}^{-1}$ variation found for the ridge material suggest the collision of gas flows separated by only $\sim 5-10$ $\mathrm{km} \mathrm{s}^{-1}$. Note that $\mathrm{SiO}$ molecules could form in the post-shock gas and thus display a factor of two lower velocities than the initial shock velocity (see Section 5.2).

Therefore, we conclude that most of the SiO emission in W43Main mainly comes from low-velocity $\left(\leqslant 10 \mathrm{~km} \mathrm{~s}^{-1}\right)$ shocks. This is convincing evidence that $\mathrm{SiO}$ molecules are formed in shock front or post-shock gas under the influence of low-velocity shocks.

\subsection{Theoretical Ability to Create SiO Molecules Through Low-velocity Shocks}

$\mathrm{SiO}$ emission has been first demonstrated to possibly arise from magnetized, stationary C-type shock models, with $v_{\text {shock }} \geqslant$ $25 \mathrm{~km} \mathrm{~s}^{-1}$ (Schilke et al. 1997; Gusdorf et al. 2008a; Guillet et al. 2007, 2011). In bipolar outflows associated with lowmass star-forming regions, for instance, Gusdorf et al. (2008b) showed that young, non-irradiated, non-stationary intermediateto-high velocity $\mathrm{C}$-shock models can also account for the $\mathrm{SiO}$ emission. On the other hand, low-velocity C-type shocks with magnetized, "irradiated" models is also proposed to explain the origin of $\mathrm{SiO}$ emission in an irradiated environment (Lesaffre et al. 2013). Guillet et al. (2009) have shown that stationary, J-type shock models also constitute reasonable candidates to generate significant amounts of $\mathrm{SiO}$ emission in molecular outflows and jets.

We examine here a few dedicated shock models, similar to models in Lesaffre et al. (2013), which demonstrate that SiO could be produced in low-velocity, $v_{\text {shock }} \leqslant 10 \mathrm{~km} \mathrm{~s}^{-1}$, shocks in W43 ridges. Figure 7(b) compares six models with different radiation fields and different fractions of $\mathrm{Si}$ in the gas phase, grain mantles, or grain cores (e.g., Lesaffre et al. 2013). Their $N_{\text {SiO }}$ predictions, as a function of the shock velocity, can directly be compared with our observations (see Figures 7(a)-(b)). Silicon, in the interstellar medium, resides mainly in the cores of silicate grains (Flower et al. 1996). Impinging particles or photons may, however, release some of the $\mathrm{Si}$ in the gas phase 
where it can potentially be oxidized by either $\mathrm{O}_{2}$ or $\mathrm{OH}$ to form the $\mathrm{SiO}$ molecule (see Le Picard et al. 2001 and references therein). In even mildly irradiated media, like, e.g., with a far-UV field of $1 G_{0}$ in units of the average interstellar radiation field and with a column density of $\sim 2 \times 10^{20} \mathrm{~cm}^{-2}$, all of these $\mathrm{SiO}$ molecules photo-dissociate and $\mathrm{Si}$ remains mostly in atomic form in the gas (Le Picard et al. 2001). As shown in Lesaffre et al. (2013), magnetized shocks, even at low-velocity, can bring enough energy to overcome the reaction barriers for $\mathrm{OH}$ and $\mathrm{O}_{2}$ formation and balance the photo-dissociation rates. These molecules are then available to oxidize $\mathrm{Si}$ and form $\mathrm{SiO}$.

The amount of $\mathrm{SiO}$ a shock can produce depends on the phase where the element $\mathrm{Si}$ is present in the pre-shock medium: grain cores, grain mantles, or gas phase. If some $\mathrm{Si}$ is already in gas phase, $\mathrm{SiO}$ can be formed at very low velocity, i.e., already around $5 \mathrm{~km} \mathrm{~s}^{-1}$ (see, e.g., solid lines in Figure 7(b)). If $\mathrm{Si}$ is present in the form of ice mantles on the grains, one needs to reach at least $\sim 10 \mathrm{~km} \mathrm{~s}^{-1}$ to sputter $\mathrm{Si}$ in the gas phase (see, e.g., dashed lines in Figure 7(b); Gusdorf et al. $2008 \mathrm{~b}$ ). If, on the other hand, all of the $\mathrm{Si}$ is locked in the core of grains, one needs to spend more energy to detach $\mathrm{Si}$, and a velocity of at least $25 \mathrm{~km} \mathrm{~s}^{-1}$ is needed (see, e.g., dotted lines in Figure 7(b); Schilke et al. 1997; Gusdorf et al. 2008a). Figure 7(b) illustrates these three scenarios in more detail for a range of C-type shock velocities using the models by Lesaffre et al. (2013). We used far-UV fields of $1 G_{0}$ (red curves) to $10 G_{0}$ (blue curves) and a density for the pre-shock gas of $n_{\mathrm{H}}=10^{5} \mathrm{~cm}^{-3}$ (see below), close to that of the W43-MM1 and W43-MM2 ridges. The magnetic field perpendicular to the shock front is another important parameter because it will determine the velocity limit at which $\mathrm{C}$ shocks cannot exist (Lesaffre et al. 2013). It is expressed through the dimensionless magnetization parameter $b=B / 1 \mu \mathrm{G} \sqrt{n_{\mathrm{H}} / 1 \mathrm{~cm}^{3}}$. We assumed a constant magnetic field strength over the shocked regions, equal to the one measured toward the W43-MM1 massive dense core: $B \sim 855 \mu \mathrm{G}$ (Cortes et al. 2010). Given the mean density of the W43-MM1 and W43-MM2 ridges listed in Table 3, we estimated $n_{\mathrm{H}}=2.33 \times n_{\mathrm{H}_{2}} \simeq 3-6 \times 10^{4} \mathrm{~cm}^{-3}$ and thus, $b \simeq 2.7-4.8$. Therefore, we set $b=3$ for our models shown in Figure 7(b).

As seen in Figure 7, the models with $\mathrm{Si}$, initially deposited in the gas or in mantles and shock velocities of $\sim 5-10 \mathrm{~km} \mathrm{~s}^{-1}$, can explain the $\mathrm{SiO}$ column density observed in the W43MM1 and W43-MM2 ridges. However, the distinction between these two types of Si reservoirs and the use of more detailed input parameters would require a multi-transition study of $\mathrm{SiO}$ emission lines incorporating other shock tracers.

In order to check the effect of the mean interstellar radiation field on the $\mathrm{SiO}$ production, we increased the far UV field strength from $G_{0}=1$ to $G_{0}=10$. We showed that the $\mathrm{SiO}$ abundance is not significantly changed (see Figure 7(b)). It proves that the observed $\mathrm{SiO}$ emission does not necessarily favor shocks in a medium highly irradiated by the UV photons of the cluster.

\subsection{Implications for the Formation of Ridge and Young Massive Cluster}

The current knowledge of high-mass star-formation points toward a dynamical picture. Accretion flows from a surrounding filamentary network (Schneider et al. 2010a; Hennemann et al. 2012) were observed and suggested to be necessary to create ridges that form the majority of high-mass stars. In principle, this process could be called "colliding flows" since they represent the collision of several gas streams. The collision observed here happens at high density $\left(n_{\mathrm{H}_{2}}>10^{3} \mathrm{~cm}^{-3}\right)$ and could be a prolongation of the converging flows process building up molecular gas from pockets of H I gas. At these densities, the cloud structures considered are gravitationally bound and even collapsing (see Table 3 and Motte et al. 2005 for W43). In colliding flows models, gravity is the main force that drives the colliding flows, sometimes called "gravitational focusing" (Burkert \& Hartmann 2004). Since the initial gravitational potential should be explained, a converging flow plus colliding stream scenario is attractive as it self-consistently forms first the cloud and then ridges. It also agrees with observations suggesting that the W43 molecular complex has been formed through large-scale colliding flows (Nguyen Luong et al. 2011b; F. Motte et al., in preparation) while harboring ridges with a coherent but complex velocity structure (see Section 3 ). The special location of W43 at the meeting point of the Galactic bar and spiral arm certainly helps to generate such dynamics (Nguyen Luong et al. 2011b; Carlhoff et al. 2013).

Theoretically, colliding flows are an efficient way to gather mass in dense cloud structures (e.g., Bergin et al. 2004; Heitsch et al. 2008; Koyama \& Inutsuka 2000). In the simple case of two streams frontally colliding, collected sheets would form and rapidly fragment into filaments (Heitsch et al. 2011). In the practical case of streams which are turbulent and/or colliding with non-zero impact angles, shears are expected to develop. Series of shocks in compressed layers should thus be natural consequences of realistic colliding flows (Bergin et al. 2001; Koyama \& Inutsuka 2000; van Loo et al. 2007). In W43-Main, the high-density ridges coincide with extended $\mathrm{SiO}$ emission, thus delineating the shocked and compressed cloud structures (see Section 3$)$. The low-velocity $\left(\leqslant 10 \mathrm{~km} \mathrm{~s}^{-1}\right)$ shocks observed along the ridges (see Section 4 ) tend to confirm that cloud ridges are formed through the gradual merging of several gas flows, as previously proposed for the DR21 ridge by Schneider et al. (2010a) and Hennemann et al. (2012). The W43-MM1 and W43MM2 immediate surroundings have $\sim 1.5$ more mass than the ridges themselves (i.e., $M_{\text {ridges }} \sim 1.5 \times 10^{4} M_{\odot}$ ) and could feed them for the next $10^{6} \mathrm{yr}$ with an infall rate close to the shock velocity found here, a few $\mathrm{km} \mathrm{s}^{-1}$.

Within the shocked filaments created by colliding flows, higher-density cloud structures/cores are expected to form, potentially leading to the formation of high-mass stars (Whitworth et al. 1994a, 1994b; van Loo et al. 2007). Ridges are large-scale, a few $\mathrm{pc}^{2}$, elongated structures with a mean density typical of protostars $\left(n_{\mathrm{H}_{2}} \sim 10^{4}-10^{5} \mathrm{~cm}^{-3}\right)$ and forming clusters of high-mass stars (Hill et al. 2011; Nguyen Luong et al. 2011a; Hennemann et al. 2012). The W43-MM1 and W43-MM2 ridges are exceptionally massive and can be qualified as candidate precursors of starburst clusters (Motte et al. 2003) or precursors of young massive clusters (Ginsburg et al. 2012). The massive dense cores identified by Motte et al. (2003) were assumed to be hosting high-mass protostars and used to estimate a star formation rate reminiscent of extragalactic starbursts: $\Sigma_{\mathrm{SFR}} \sim 1000 M_{\odot} \mathrm{yr}^{-1} \mathrm{kpc}^{-2}$. While the three most massive dense cores (W43-MM1, W43-MM2, and W43MM3 with $M=1000-4000 M_{\odot}$ within $\sim 0.2$ pc sizes) are clearly forming one/a few high-mass stars, the exact nature of the others is still unclear. One needs higher-angular observations to search for high-mass protostars in the W43-MM1 and W43-MM2 ridges and confirm that the star formation rates of these ridges are high (F. Louvet et al., in preparation). Such a 
result is consistent with the high star formation activity measured toward other ridges studied with Herschel (e.g., Nguyen Luong et al. 2011b; M. Hennemann et al., in preparation). Note that, in contrast, the CMZ presents high-density clouds that may be devoid of high-mass star formation (e.g., Menten et al. 2009). If confirmed, such a low star formation activity could be related to the large strength of the velocity shears in the CMZ. Thus, the W43-MM1 and W43-MM2 ridges are high-density cloud structures forming from constructive low-velocity colliding flows while other regions, like CMZ, might suffer from destructive colliding flows or strong radiation fields.

\section{CONCLUSION AND SUMMARY}

We report the discovery of two ridge structures and extended $\mathrm{SiO}$ emission in the W43-Main mini-starburst region. We used a combination of Herschel continuum data in the 160-350 $\mu \mathrm{m}$ wavebands and IRAM $30 \mathrm{~m}$ telescope data of the $\mathrm{SiO}(2-1)$ and $\mathrm{N}_{2} \mathrm{H}^{+}(1-0)$ emission lines (see Tables 1 and 2). Our main findings can be summarized as follows.

1. On the Herschel column density image, W43-Main harbors two high-column density $\left(N_{\mathrm{H}_{2}}>10^{23} \mathrm{~cm}^{-2}\right)$ structures, which we call the W43-MM1 and W43-MM2 ridges (see Figure 2(a)).

2. Above a background level of $4 \times 10^{22} \mathrm{~cm}^{-2}$, the W43MM1 and W43-MM2 ridges have masses of 21,000 $M_{\odot}$ and $35,000 M_{\odot}$ within 6 and $14 \mathrm{pc}^{2}$ areas, respectively (see Table 3). The corresponding volume and surface densities are $n_{\mathrm{H}_{2}} \sim 6 \times 10^{4} \mathrm{~cm}^{-3}$ and $\Sigma_{S}=3500 M_{\odot} \mathrm{pc}^{-2}$ for W43-MM1 ridge or $n_{\mathrm{H}_{2}} \sim 3.4 \times 10^{4} \mathrm{~cm}^{-3}$ and $\Sigma_{S}=$ $2300 M_{\odot} \mathrm{pc}^{-2}$ for $\mathrm{W} 43-\mathrm{MM} 2$ ridge.

3. The immediate surroundings of the W43-MM1 and W43MM2 ridges, defined as cloud structures above $4 \times$ $10^{22} \mathrm{~cm}^{-2}$, have a mean density of $n_{\mathrm{H}_{2}} \sim 5 \times 10^{3} \mathrm{~cm}^{-3}$ (see Figure 2(a) and Table 3). They provide a mass reservoir of 32,000 $M_{\odot}$ for the W43-MM1 ridge and 53,000 $M_{\odot}$ for the W43-MM2 ridge that could be used to further build up the ridges and enhance its subsequent star formation.

4. The $\mathrm{N}_{2} \mathrm{H}^{+}$(1-0) spectra measured toward the W43MM1 and W43-MM2 ridges (see Figure 3 and Table 4) suggest that these dense cloud structures are complex, with velocities spanning the $V_{\mathrm{LSR}} \sim 94-97 \mathrm{~km} \mathrm{~s}^{-1}$ and $88-95 \mathrm{~km} \mathrm{~s}^{-1}$ ranges, respectively. The W43-MM1 and W43-MM2 ridges, however, are coherent velocity structures and despite their large $\mathrm{N}_{2} \mathrm{H}^{+}$velocity dispersion, $\sigma \sim 2 \mathrm{~km} \mathrm{~s}^{-1}$, are gravitationally bound.

5. In W43-Main, the $\mathrm{SiO}(2-1)$ emission spreads over two large areas: $\sim 12 \mathrm{pc}^{2}$ and $\sim 16 \mathrm{pc}^{2}$ (see Figure 5). They cover the W43-MM1 and W43-MM2 ridges and part of their immediate surroundings. This $\mathrm{SiO}$ emission is, with a few clouds in the CMZ, among the most extended observed up to now in our Galaxy.

6. The $\mathrm{SiO}(2-1)$ spectra observed along the ridges and integrated over the entire W43-Main (see Figures 4 and 5) can correctly be described by single Gaussian velocity dispersions $\left(\sigma \sim 2.5-5.2 \mathrm{~km} \mathrm{~s}^{-1}\right)$ without any obvious wings (FWZP $<25 \mathrm{~km} \mathrm{~s}^{-1}$; see Table 5). SiO (2-1) lines are also peaking at the same velocities as the $\mathrm{N}_{2} \mathrm{H}^{+}(1-0)$ and ${ }^{13} \mathrm{CO}(1-0)$ lines. The absence of high-velocity $\mathrm{SiO}$ wing emission in W43 is consistent with not having $\mathrm{SiO}$ arising from outflow shocks, as observed in other starforming regions.
7. Despite the $\sim 0.9$ pc resolution of our $\mathrm{SiO}$ (2-1) map, the lines are bright (1-11 $\mathrm{K} \mathrm{km} \mathrm{s}^{-1}$; see Figure 5), roughly corresponding to a $\mathrm{SiO}$ column density of $N_{\mathrm{SiO}} \sim$ $6 \times 10^{13} \mathrm{~cm}^{-2}$. The luminosities of $31^{\prime \prime}$ beam-pixels in the W43-MM1 and W43-MM2 regions are $L_{\mathrm{SiO}(2-1)} \sim$ 200-2700 $\mathrm{K} \mathrm{km} \mathrm{s}^{-1} \mathrm{kpc}^{2}$, summing up to a total luminosity of $L_{\mathrm{SiO}(2-1)}^{\mathrm{total}} \sim 4 \times 10^{4}$ or $\sim 2 \times 10^{4} \mathrm{~K} \mathrm{~km} \mathrm{~s}^{-1} \mathrm{kpc}^{2}$ for each of the ridges.

8. The $L-F W Z P$ relation is steeper for the $\mathrm{SiO}$ lines arising from W43-Main than for most $\mathrm{SiO}$ lines detected toward comparative samples of massive young stellar objects (see Figure 5 and Table 5). We propose the use of such diagrams and slopes of the linear fits to distinguish between lowvelocity $\left(\leqslant 10 \mathrm{~km} \mathrm{~s}^{-1}\right)$ and high-velocity $\left(20-100 \mathrm{~km} \mathrm{~s}^{-1}\right)$ shocks, for both observational studies and synthetic observations of shock models.

9. Small offsets in velocity and positions $\left(\sim 0.8 \mathrm{~km} \mathrm{~s}^{-1}\right.$ and $\leqslant 0.5 \mathrm{pc}$; see Table 4 and Figure 5) are measured between the $\mathrm{SiO}$ and $\mathrm{N}_{2} \mathrm{H}^{+}$lines observed along the W43-MM1 ridge. They recall the relative motions expected between post- and pre-shocked gases.

10. Dedicated shock models prove that low-velocity $\left(5-10 \mathrm{~km} \mathrm{~s}^{-1}\right)$ shocks could give rise to bright and extended $\mathrm{SiO}$ emission, provided that $10 \% \mathrm{Si}$ is initially deposited in the gas phase or in the grain ice mantles (see Figure 7). The exact process responsible for $\mathrm{SiO}$ emission in the W43-MM1 and W43-MM2 ridges is surely not grain core destruction as advocated for high-velocity $\left(>20 \mathrm{~km} \mathrm{~s}^{-1}\right)$ shocks developing along protostellar outflows. Among the main open questions are how $\mathrm{Si}$ could exist in the gas phase or in grain mantles and with which abundance.

11. The structure and kinematics of the W43-MM1 and W43MM2 ridges are tightly linked to those of the shocked gas observed in SiO. Low-velocity $\left(\leqslant 10 \mathrm{~km} \mathrm{~s}^{-1}\right)$ shocks may thus indicate that shears have developed when collisioning several gas flows arising from the ridge immediate surroundings. This interpretation is consistent with the idea that cloud ridges (and their embedded massive dense cores) form through colliding flows or merging of filaments.

Part of this work was supported by the ANR (Agence Nationale pour la Recherche) projects "PROBeS" (number: ANR-08-BLAN-0241) and "STARFICH" (number: ANR-11BS56-010). A. Gusdorf acknowledges support by the grant ANR-09-BLAN-0231-01 from the French Agence Nationale de la Recherche as part of the SCHISM project. L.B. acknowledges support from CONICYT project PFB-06. We thank Sergio Molinari for giving us permission to use the Herschel images and the IRAM staff for their wonderful hospitality and observing services. This work has benefited from research funding from the European Community's Seventh Framework Programme.

\section{APPENDIX A \\ ONLINE IMAGES}

In Figure 8, we show the composite three-color Herschel image of the W43 molecular cloud complex (70 $\mu \mathrm{m}$ : blue, $160 \mu \mathrm{m}$ : green, $250 \mu \mathrm{m}$ : red). The column density and the dust temperature maps of the entire W43 molecular cloud complex can be seen in Figure 9. 


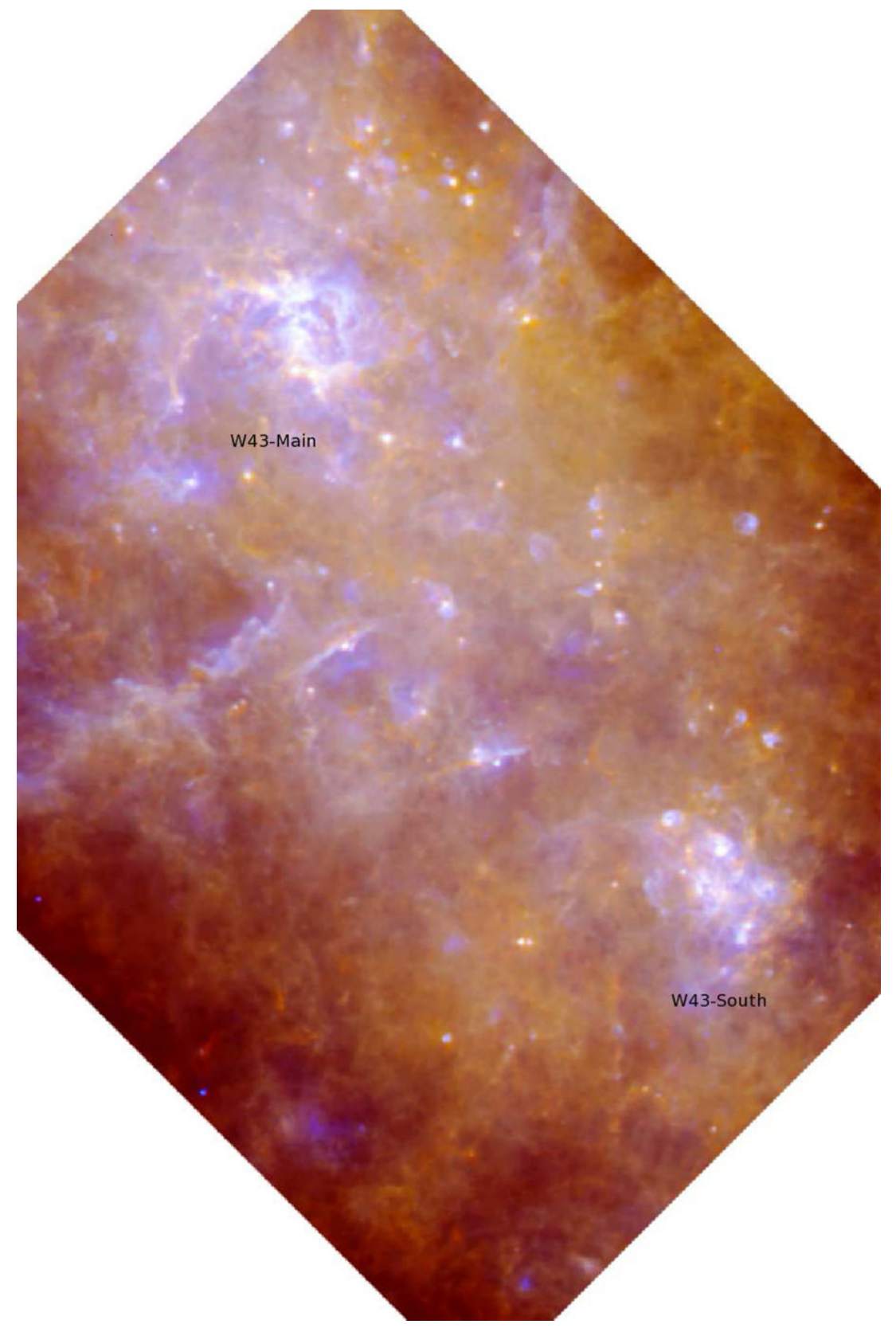

Figure 8. Composite three-color Herschel image of the W43 molecular cloud complex (70 $\mu$ m: blue, $160 \mu \mathrm{m}$ : green, $250 \mu \mathrm{m}$ : red). The image is oriented in right ascension and declination directions while the complex was defined by a rectangle in Galactic coordinates (see Nguyen Luong et al. 2011a). The blue component traces $\mathrm{H}$ II and photon-dominated regions while earlier stage star-forming sites, such as cores and filaments, are traced by the red component.

(A color version of this figure is available in the online journal.)

\section{APPENDIX B \\ SATURATION CORRECTION OF HERSCHEL/SPIRE IMAGES}

We explain here the process developed to correct for the saturation of SPIRE images, presented in Section 2.1 and used to build the column density and dust temperature images (see Section 3.1). This process has already been successfully used to correct the bright-mode SPIRE images of the HOBYS Key program (e.g., Rivera-Ingraham et al. 2013; Fallscheer et al. 2013). In the case of W43, the Hi-GAL observations were performed in the nominal voltage bias mode, and as a result, the SPIRE detectors were saturating on bright sources, i.e., the inner parts of W43-Main and W43-South. We used SPIRE data taken in the bright-source voltage bias mode, as part of the HOBYS project (see Section 2.1), to replace the Hi-GAL map pixels suffering from soft saturation (see definition in Appendix B.1) or which are missing. To this end, the HOBYS data were processed through the exact same steps as those used for the Hi-GAL data, including mapping into the same coordinate system using Scanamorphos (see Section 2.1 and Figures 10(a)-(f)). 

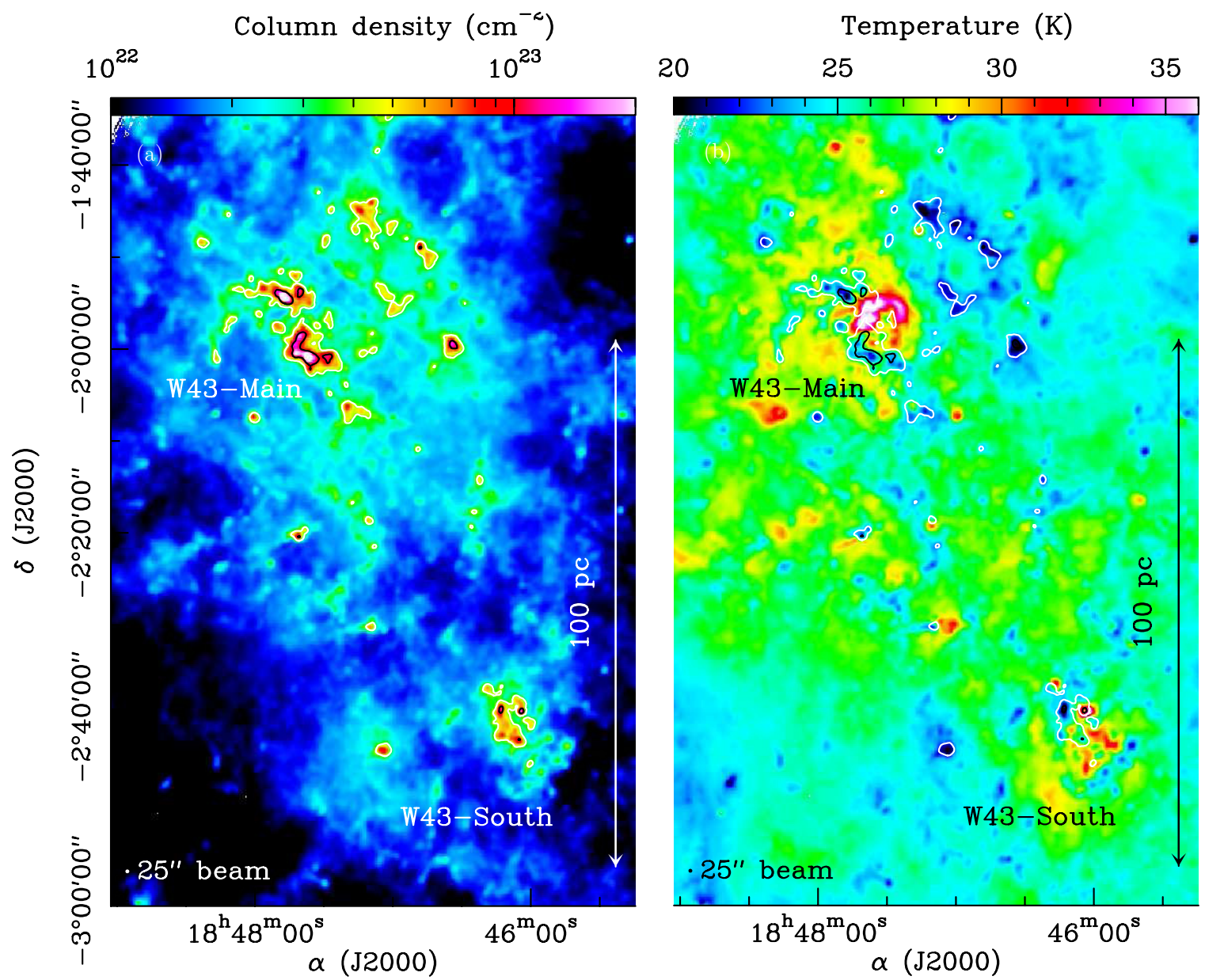

Figure 9. W43 molecular complex in: (a) Herschel column density (color and contours), and (b) dust temperature (color) and column density (contours). The white and black contours, corresponding to the $4 \times 10^{22} \mathrm{~cm}^{-2}$ and $1 \times 10^{23} \mathrm{~cm}^{-2}$ levels, are outlining the W43-MM1 and W43-MM2 ridges and their immediate surroundings boundary.

(A color version of this figure is available in the online journal.)

\section{B.1. Determination of the "Soft" Saturation Limit}

In Figure 11, we plotted the signal and weight maps of the region around W43-Main as observed by the Hi-GAL and HOBYS surveys (top and bottom rows, respectively). Saturated map pixels were flagged as $\mathrm{NaN}$ when all bolometers passing over the regions produced signals that fell out the range of the electronics. These pixels are shown in white and are located over the W43-MM1, W43-MM2, and W43-MM3 massive dense cores. The astronomical surface brightness at which this occurs varies from bolometer to bolometer as it depends on how the voltage offset for the detector signal was set at the beginning of the observation. In practice, a bolometer with a baseline signal near the high signal limit saturates more easily.

Furthermore, the map pixels near the bright saturated regions have a non-linear response to the incoming flux. Bolometers traveling up or down the bright sources produced usable data only at the faint beginning and end of their measurements. In this case, the signal values of the map pixels were weighted down with increasing surface brightness, making signals close to bright sources no longer reliable. This is illustrated by the sudden decrease in the weight map toward the centers of the W43-MM1, W43-MM2, and W43-MM3 massive dense cores (see Figure 11, upper right). Therefore, it is also necessary to replace the signal in these "softly" saturated pixels. We visually checked both the weight and signal maps around the $\mathrm{NaN}$ pixels and determined that map pixels with fluxes larger than $\sim 150 \mathrm{Jy} \mathrm{beam}^{-1}$, for all Herschel wavebands, are significantly affected by saturation effects. This threshold value is also consistent with the surface brightness above which individual bolometers of the SPIRE arrays were saturated in flux calibration data (Bendo et al. 2013).

\section{B.2. Offset Addition to the Bright-source-mode Maps and Replacement of Saturated Pixels in the Nominal-mode Maps}

Before replacing the saturated pixels in the nominal voltage bias mode (Hi-GAL) SPIRE maps with those from the brightsource voltage bias mode (HOBYS) maps, we added offsets to the bright-mode map pixels. To derive such offsets, we first masked the region surrounding the $\mathrm{NaN}$ pixels, then correlated surface brightness pixel by pixel over the common areas where Hi-GAL pixels are not saturated (see Figures 12(a)-(c)). The correlation weakens as the surface brightness increases, especially above the "soft" saturation limit of $150 \mathrm{Jy}^{\text {beam }}{ }^{-1}$. Elsewhere, both datasets are linearly correlated, as shown in Figures 12(a)-(c). The pixels with fluxes $>150 \mathrm{Jy}^{-1}$ beam $^{-1}$ in the Hi-GAL maps, i.e., the saturated pixels and those with 


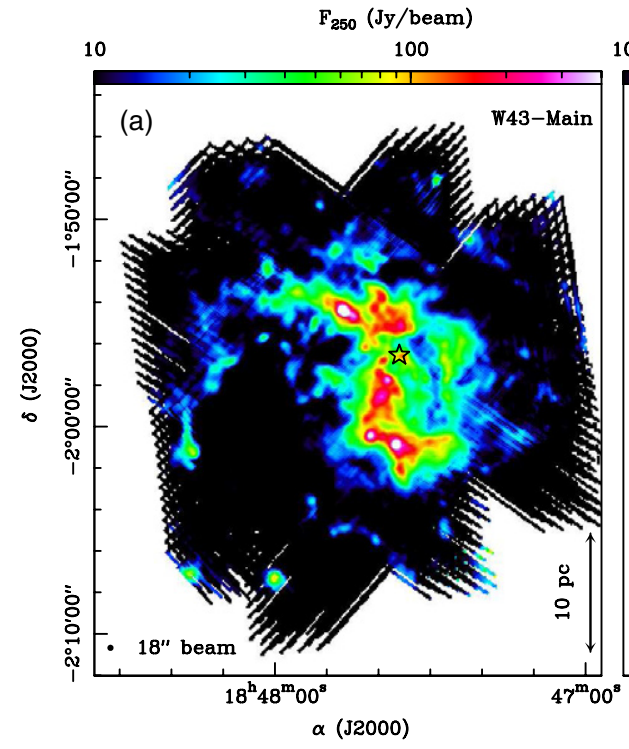
10
$\mathrm{F}_{350}(\mathrm{Jy} /$ beam $)$ 100
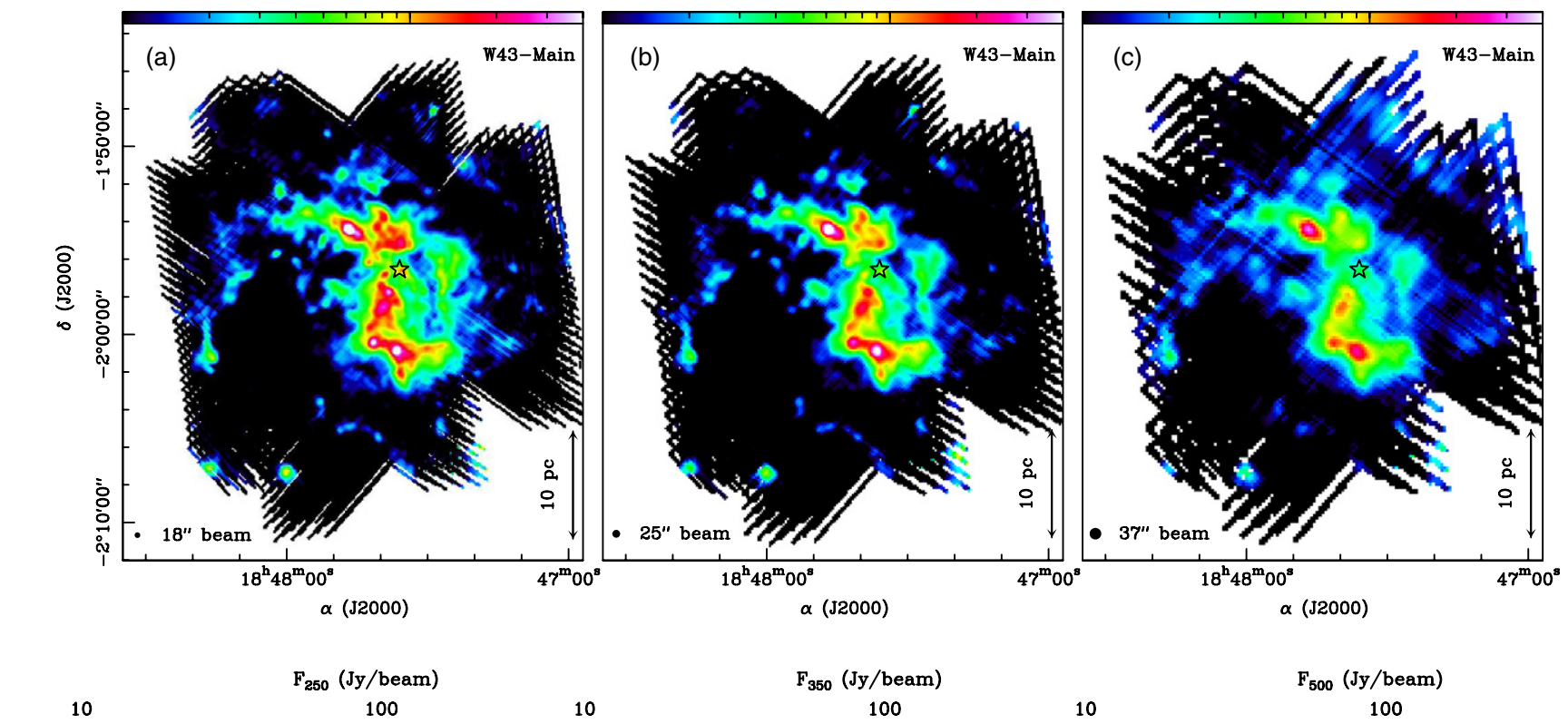

10

$\mathrm{F}_{350}(\mathrm{Jy} /$ beam $)$

100
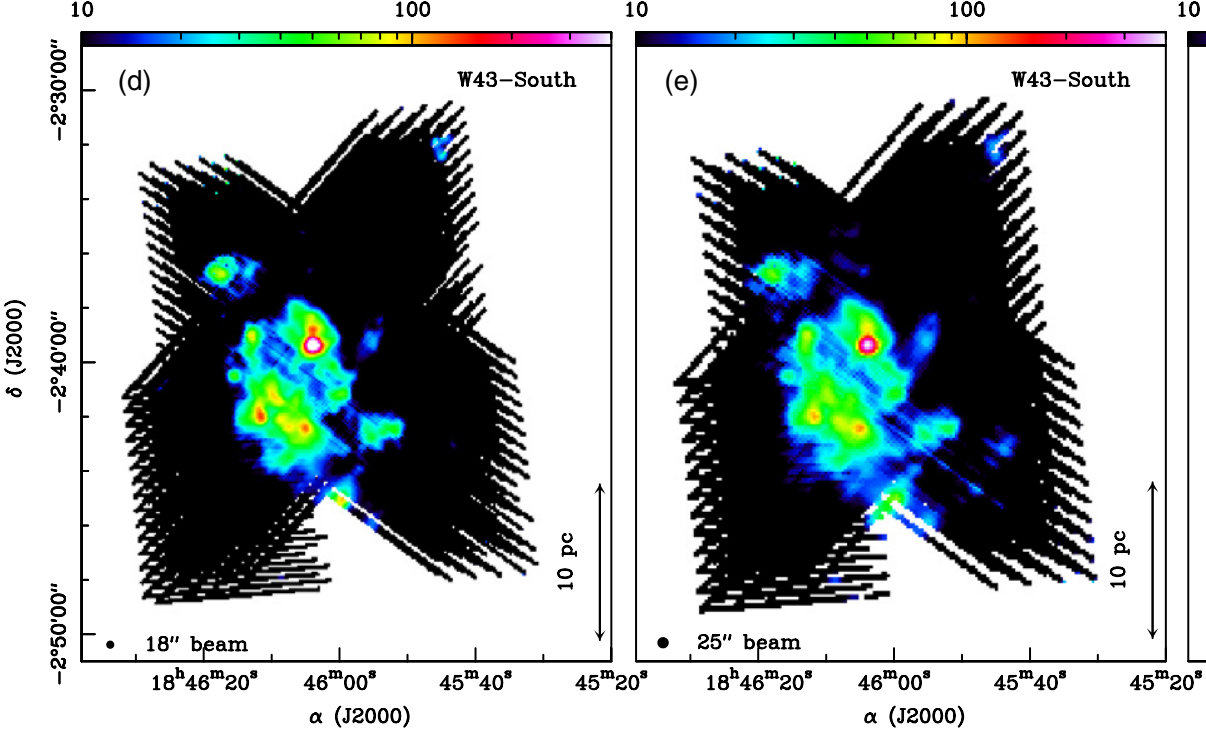

10

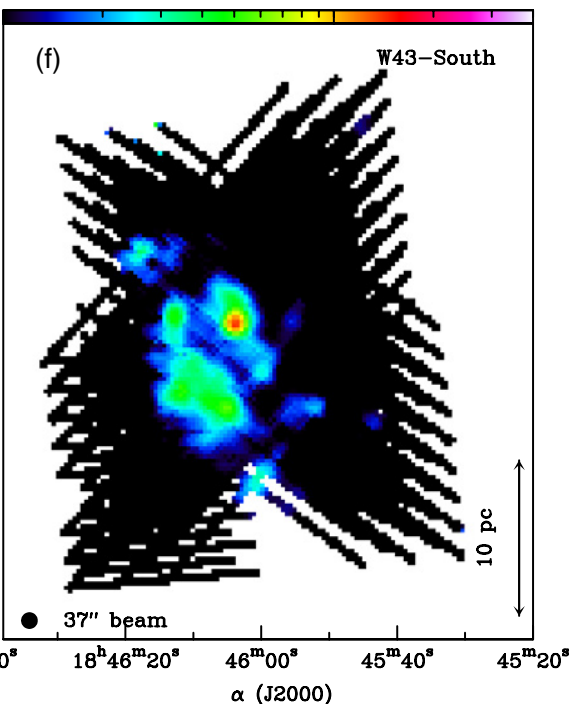

Figure 10. W43-Main (upper row) and W43-South (lower row) regions as observed by the HOBYS key program at 250,350 , and $500 \mu \mathrm{m}$ Herschel wavelengths.

(A color version of this figure is available in the online journal.)

non-linear response, were then replaced by the ones from the HOBYS maps with offsets. As can be seen in Figures 13(a)-(c), the repaired maps better correlate with the bright-mode maps. The resulting image presents a smooth transition from the pixels initially coming from the Hi-GAL dataset and the HOBYS dataset with offsets added.

\section{APPENDIX C}

\section{CONVERSION THE UNIT OF LINE LUMINOSITY FROM $\mathrm{K} \mathrm{km} \mathrm{s}^{-1} \mathrm{kpc}^{2}$ INTO SOLAR LUMINOSITY $L_{\odot}$}

The unit $\mathrm{K} \mathrm{km} \mathrm{s}^{-1} \mathrm{kpc}^{2}$ of the molecular line luminosity calculated from Equation (6) can be expressed also in the unit of the sun bolometric luminosity $L_{\odot}$. One $L_{\odot}$ equals $3.846 \times 10^{26}$ $\mathrm{W}$, which can be converted into the Jy unit of flux density as $1 L_{\odot}=3.846 \times 10^{26} \mathrm{~W}=3.846 \times 10^{52} \mathrm{Jy} \mathrm{Hz} \mathrm{m}^{2}=4 \times 10^{13}$ $\mathrm{Jy} \mathrm{Hz} \mathrm{kpc}{ }^{2}$. The frequency-integrated flux density unit $\mathrm{Jy} \mathrm{Hz}$ is equivalent to the velocity-integrated main-beam temperature $\mathrm{K}$ $\mathrm{km} \mathrm{s}^{-1}$, the conversion between the two units is as following

$$
\frac{\int F_{\nu} d v}{\mathrm{Jy} \mathrm{Hz}}=8.17 \times 10^{-7} \frac{v_{0}^{2}}{\mathrm{GHz}^{2}} \frac{\theta^{2}}{{ }^{\prime 2}} \frac{\int T_{\mathrm{mb}} d v}{\mathrm{Kkm} \mathrm{s}^{-1}} \frac{v_{0}}{\mathrm{~Hz}} \frac{c^{-1}}{\left(\mathrm{~km} \mathrm{~s}^{-1}\right)^{-1}} \text {. }
$$

Thus, for our $\mathrm{SiO} 2-1$ observation, the conversion between the line luminosity in unit of $\mathrm{K} \mathrm{km} \mathrm{s}^{-1} \mathrm{kpc}^{2}$ to the unit of the sun bolometric luminosity $L_{\odot}$ is

$$
\begin{aligned}
\frac{L_{\mathrm{SiO} 2-1} L_{\odot}=}{} & 8.17 \times 10^{-7} \times \frac{L_{\mathrm{SiO} 2-1}}{\mathrm{Kkm} \mathrm{s}^{-1} \mathrm{kpc}^{2}} \frac{v_{0}^{2}}{\mathrm{GHz}^{2}} \frac{\theta^{2}}{{ }^{\prime \prime}} \frac{v_{0}}{\mathrm{~Hz}} \\
& \times \frac{c^{-1}}{\left(\mathrm{~km} \mathrm{~s}^{-1}\right)^{-1}} \times \frac{1}{4 \times 10^{13}}=4.28 \times 10^{-8} \\
& \times \frac{L_{\mathrm{SiO} 2-1}}{\mathrm{~K} \mathrm{~km} \mathrm{~s}^{-1} \mathrm{kpc}^{2}}
\end{aligned}
$$



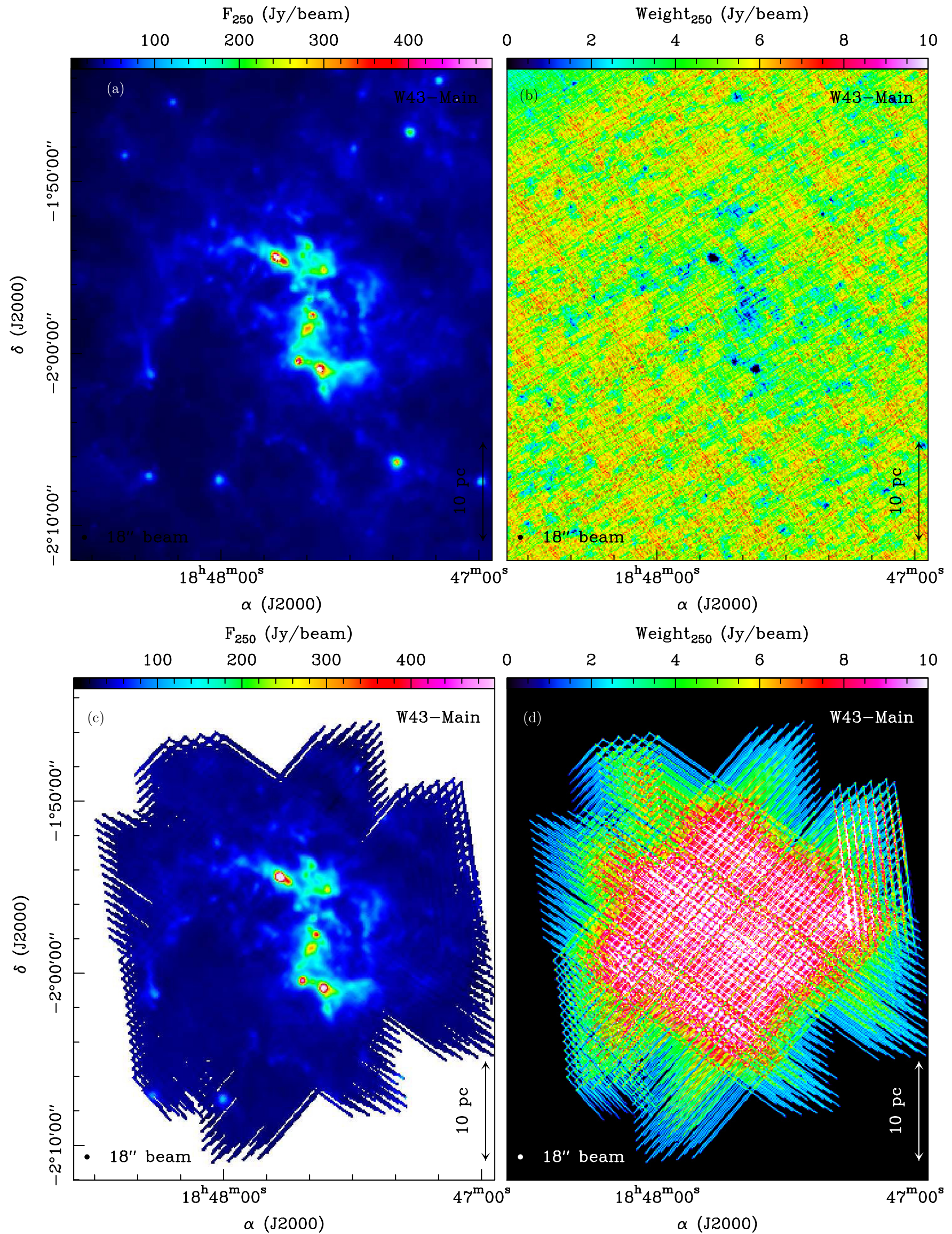

Figure 11. Herschel $250 \mu \mathrm{m}$ images of W43-Main taken from the Hi-GAL in (a) and HOBYS in (c) key programs. Their weight maps are plotted in (b) and (d), respectively.

(A color version of this figure is available in the online journal.) 

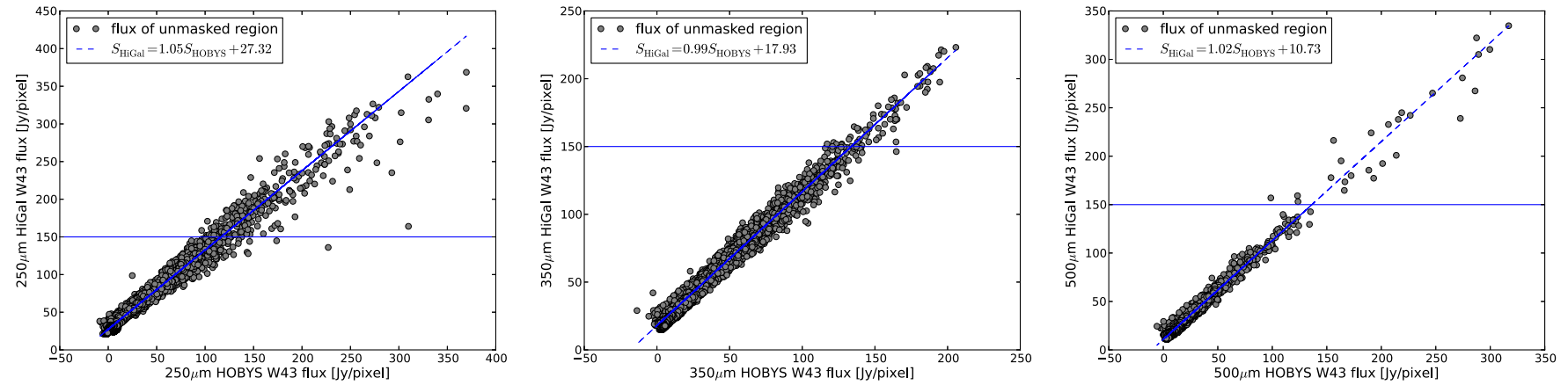

Figure 12. The pixel-by-pixel correlation between initial Hi-GAL and HOBYS datasets over the unsaturated common regions.
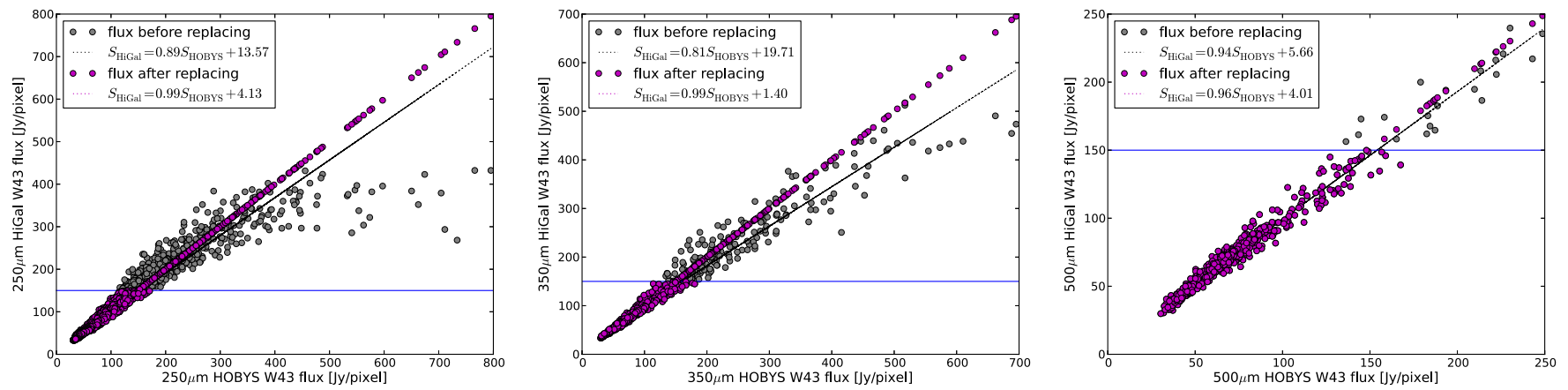

Figure 13. Pixel-by-pixel correlation between Hi-GAL and HOBYS+offset datasets before (green circles) and after saturation correction (pink circles).

\section{REFERENCES}

Acord, J. M., Walmsley, C. M., \& Churchwell, E. 1997, ApJ, 475, 693

Ballesteros-Paredes, J., Hartmann, L., \& Vázquez-Semadeni, E. 1999, ApJ, 527, 285

Bally, J., Anderson, L. D., Battersby, C., et al. 2010, A\&A, 518, L90

Bendo, G. J., Griffin, M. J., Bock, J. J., et al. 2013, MNRAS, 433, 3062

Bergin, E. A., Ciardi, D. R., Lada, C. J., Alves, J., \& Lada, E. A. 2001, ApJ, 557, 209

Bergin, E. A., Hartmann, L. W., Raymond, J. C., \& Ballesteros-Paredes, J. 2004, ApJ, 612, 921

Beuther, H., Schilke, P., Sridharan, T. K., et al. 2002, A\&A, 383, 892

Beuther, H., \& Sridharan, T. K. 2007, ApJ, 668, 348

Bieging, J. H., Peters, W. L., \& Kang, M. 2010, ApJS, 191, 232

Bik, A., Kaper, L., Hanson, M. M., \& Smits, M. 2005, A\&A, 440, 121

Blum, R. D., Damineli, A., \& Conti, P. S. 1999, AJ, 117, 1392

Bonnell, I. A., \& Bate, M. R. 2006, MNRAS, 370, 488

Bressert, E., Ginsburg, A., Bally, J., et al. 2012, ApJL, 758, L28

Burkert, A., \& Hartmann, L. 2004, ApJ, 616, 288

Carlhoff, P., Nguyen Luong, Q., Schilke, P., et al. 2013, A\&A, in press (arXiv:1308.6112)

Chièze, J.-P., Pineau des Forets, G., \& Flower, D. R. 1998, MNRAS, 295, 672

Cortes, P. C., Parra, R., Cortes, J. R., \& Hardy, E. 2010, A\&A, 519, A35

Csengeri, T., Bontemps, S., Schneider, N., Motte, F., \& Dib, S. 2011a, A\&A, 527, A 135

Csengeri, T., Bontemps, S., Schneider, N., et al. 2011b, ApJL, 740, L5

Draine, B. T. 1980, ApJ, 241, 1021

Duarte-Cabral, A., Bontemps, S., Motte, F., et al. 2013, A\&A, in press (arXiv:1308.6490)

Fallscheer, C., Reid, M. A., Di Francesco, J., et al. 2013, ApJ, 773, 102

Flower, D. R., Pineau des Forets, G., Field, D., \& May, P. W. 1996, MNRAS, 280, 447

Galván-Madrid, R. 2013, ApJ, submitted

Ginsburg, A., Bressert, E., Bally, J., \& Battersby, C. 2012, ApJL, 758, L29

Griffin, M. J., Abergel, A., Abreu, A., et al. 2010, A\&A, 518, L3

Gueth, F., Guilloteau, S., \& Bachiller, R. 1998, A\&A, 333, 287

Guillet, V., Jones, A. P., \& Pineau Des Forêts, G. 2009, A\&A, 497, 145

Guillet, V., Pineau Des Forêts, G., \& Jones, A. P. 2007, A\&A, 476, 263

Guillet, V., Pineau Des Forêts, G., \& Jones, A. P. 2011, A\&A, 527, A123

Gusdorf, A., Cabrit, S., Flower, D. R., \& Pineau Des Forêts, G. 2008a, A\&A, 482,809

Gusdorf, A., Pineau Des Forêts, G., Cabrit, S., \& Flower, D. R. 2008b, A\&A, 490,695
Güsten, R., \& Philipp, S. D. 2004, in The Dense Interstellar Medium in Galaxies, ed. S. Pfalzner, C. Kramer, C. Staubmeier, \& A. Heithausen (Berlin: Springer), 253

Hartmann, L., Ballesteros-Paredes, J., \& Bergin, E. A. 2001, ApJ, 562, 852

Hartmann, L., Ballesteros-Paredes, J., \& Heitsch, F. 2012, MNRAS, 420, 1457

Hartmann, L., \& Burkert, A. 2007, ApJ, 654, 988

Hatchell, J., Fuller, G. A., \& Millar, T. J. 2001, A\&A, 372, 281

Heitsch, F., \& Hartmann, L. 2008, ApJ, 689, 290

Heitsch, F., Hartmann, L. W., \& Burkert, A. 2008, ApJ, 683, 786

Heitsch, F., Naab, T., \& Walch, S. 2011, MNRAS, 415, 271

Hennemann, M., Motte, F., Schneider, N., et al. 2012, A\&A, 543, L3

Henshaw, J. D., Caselli, P., Fontani, F., et al. 2013, MNRAS, 428, 3425

Hildebrand, R. H. 1983, QJRAS, 24, 267

Hill, T., André, P., Arzoumanian, D., et al. 2012, A\&A, 548, L6

Hill, T., Motte, F., Didelon, P., et al. 2011, A\&A, 533, A94

Hosokawa, T., \& Omukai, K. 2009, ApJ, 691, 823

Inoue, T., \& Fukui, Y. 2013, ApJL, submitted (arXiv:1305.4655)

Inoue, T., \& Inutsuka, S.-i. 2009, ApJ, 704, 161

Jiménez-Serra, I., Caselli, P., Tan, J. C., et al. 2010, MNRAS, 406, 187

Jones, P. A., Burton, M. G., Cunningham, M. R., et al. 2012, MNRAS, 419, 2961

Jørgensen, J. K., Bourke, T. L., Nguyen Luong, Q., \& Takakuwa, S. 2011, A\&A, 534, A100

Kauffmann, J., Pillai, T., \& Zhang, Q. 2013, ApJL, 765, L35

Koyama, H., \& Inutsuka, S. 2000, ApJ, 532, 980

Kruijssen, J. M. D., Longmore, S. N., Elmegreen, B. G., et al. 2013, MNRAS, submitted (arXiv:1303.6286)

Krumholz, M. R., \& Matzner, C. D. 2009, ApJ, 703, 1352

Krumholz, M. R., McKee, C. F., \& Tumlinson, J. 2009, ApJ, 693, 216

Le Picard, S. D., Canosa, A., Pineau des Forêts, G., Rebrion-Rowe, C., \& Rowe, B. R. 2001, A\&A, 372, 1064

Lefloch, B., Castets, A., Cernicharo, J., \& Loinard, L. 1998, ApJL, 504, L109

Lesaffre, P., Pineau des Forêts, G., Godard, B., et al. 2013, A\&A, 550, A106

López-Sepulcre, A., Walmsley, C. M., Cesaroni, R., et al. 2011, A\&A, 526, L2

Martin-Pintado, J., de Vicente, P., Fuente, A., \& Planesas, P. 1997, ApJL, 482, L45

Meixner, M., \& Tielens, A. G. G. M. 1993, ApJ, 405, 216

Menten, K. M., Wilson, R. W., Leurini, S., \& Schilke, P. 2009, ApJ, 692, 47

Molinari, S., Swinyard, B., Bally, J., et al. 2010, A\&A, 518, L100

Motte, F., Bontemps, S., Hennemann, M., et al. 2012, in SF2A-2012: Proceedings of the Annual meeting of the French Society of Astronomy and Astrophysics, ed. S. Boissier, P. de Laverny, N. Nardetto, R. Samadi, D. Valls-Gabaud, \& H. Wozniak, 45 
Motte, F., Bontemps, S., Schilke, P., et al. 2005, in IAU Symp. 227, Massive Star Birth: A Crossroads of Astrophysics, ed. R. Cesaroni, M. Felli, E. Churchwell, \& M. Walmsley (Cambridge: Cambridge Univ. Press), 151

Motte, F., Bontemps, S., Schilke, P., et al. 2007, A\&A, 476, 1243

Motte, F., Schilke, P., \& Lis, D. C. 2003, ApJ, 582, 277

Motte, F., Zavagno, A., Bontemps, S., et al. 2010, A\&A, 518, L77

Müller, H. S. P., Schlöder, F., Stutzki, J., \& Winnewisser, G. 2005, JMoSt, 742, 215

Nguyen Luong, Q., Motte, F., Hennemann, M., et al. 2011a, A\&A, 535, A76

Nguyen Luong, Q., Motte, F., Schuller, F., et al. 2011b, A\&A, 529, A41

Poglitsch, A., Waelkens, C., Geis, N., et al. 2010, A\&A, 518, L2

Requena-Torres, M. A., Martín-Pintado, J., Rodríguez-Franco, A., et al. 2006, A\&A, 455, 971

Rivera-Ingraham, A., Martin, P. G., Polychroni, D., et al. 2013, ApJ, 766, 85

Rodríguez, L. F., \& Zapata, L. A. 2013, ApJL, 767, L13

Rohlfs, K., \& Wilson, T. L. 2000, Tools of Radio Astronomy (New York: Springer)
Roussel, H. 2012, arXiv:1205.2576

Schilke, P., Benford, D. J., Hunter, T. R., Lis, D. C., \& Phillips, T. G. 2001, ApJS, 132,281

Schilke, P., Groesbeck, T. D., Blake, G. A., \& Phillips, T. G. 1997, ApJS, 108,301

Schneider, N., Csengeri, T., Bontemps, S., et al. 2010a, A\&A, 520, A49

Schneider, N., Csengeri, T., Hennemann, M., et al. 2012, A\&A, 540, L11

Schneider, N., Motte, F., Bontemps, S., et al. 2010b, A\&A, 518, L83

Smith, L. F., Biermann, P., \& Mezger, P. G. 1978, A\&A, 66, 65

van Loo, S., Falle, S. A. E. G., Hartquist, T. W., \& Moore, T. J. T. 2007, A\&A, 471,213

Watt, S., \& Mundy, L. G. 1999, ApJS, 125, 143

Whitworth, A. P., Bhattal, A. S., Chapman, S. J., Disney, M. J., \& Turner, J. A. 1994a, A\&A, 290, 421

Whitworth, A. P., Bhattal, A. S., Chapman, S. J., Disney, M. J., \& Turner, J. A. 1994b, MNRAS, 268, 291

Yusef-Zadeh, F., Royster, M., Wardle, M., et al. 2013, ApJL, 767, L32 